\title{
A RATIO OF INTEGRATION BETWEEN QUOTIENTS IN GEOMETRIC INVARIANT THEORY
}

\author{
ZACHARY MADDOCK
}

\begin{abstract}
Let $T$ be a maximal torus of a connected reductive group $G$ that acts linearly on a projective variety $X$ so that all semi-stable points are stable. This paper compares the integration on the geometric invariant theory quotient $X / / G$ of Chow classes $\sigma$ to the integration on the geometric invariant theory quotient $X / / T$ of certain lifts of $\sigma$ twisted by $c_{\text {top }}(\mathfrak{g} / \mathfrak{t})$, the top Chern class of the $T$-equivariant vector bundle induced by the quotient of the adjoint representation on the Lie algebra of $G$ by that of $T$. We provide a purely algebraic proof that the ratio between any two such integrals is an invariant of the group $G$ and that it equals the order of the Weyl group whenever the root system of $G$ decomposes into irreducible root systems of type $\mathbf{A}_{n}$, for various $n \in \mathbb{N}$. As a corollary, we are able to remove this restriction on root systems by applying a related result of Martin from symplectic geometry.
\end{abstract}

\section{INTRODUCTION}

A brief history. The cohomology of quotients arising from geometric invariant theory (GIT) has been the object of extensive study. In 1984, Kirwan [22] integrated the previous works of Hesselink, Kempf and Ness [15, 16, 20, 21] to explore the structure of GIT quotients from both the algebraic and symplectic perspectives, ultimately finding formulas to compute Hodge numbers. Five years later, Ellingsrud and Strømme [10] began to study the relationship between the Chow rings of the two GIT quotients $\mathbb{P}_{\bar{k}}^{n} / / G$ and $\mathbb{P}_{\bar{k}}^{n} / / T$, for a reductive group $G$ over an algebraically closed field $\bar{k}$ with maximal torus $T \subseteq G$ acting on $\mathbb{P}_{\bar{k}}^{n}$ so that all semi-stable points have trivial stabilizers; their main result was a presentation of the Chow ring $A^{*}\left(\mathbb{P}^{n} / / G\right)_{\mathbb{Q}}$ in terms of explicit generators and relations. Brion [3] then expanded this relationship to arbitrary linear actions of connected reductive groups $G$ on smooth, projective varieties $X$ over the complex numbers, proving that the $G$-equivariant cohomology of the locus of $G$-semi-stable points is isomorphic to the subgroup of Weyl anti-invariant classes of the $T$-equivariant cohomology group of the larger locus of $T$-semistable points:

$$
\phi: H_{G}^{*}\left(X_{G}^{s s} ; \mathbb{Q}\right) \stackrel{\cong}{\rightrightarrows} H_{T}^{*}\left(X_{T}^{s s} ; \mathbb{Q}\right)^{a} .
$$

Later Brion and Joshua [5] extended these results further to the case of singular $X$, but with equivariant intersection cohomology used as a suitable replacement for the standard theory.

Brion's construction of the isomorphism $\phi$ is as follows (see [3] for full details). As $X_{G}^{s s}$ is a $G$ variety, the inclusion $T \subseteq G$ induces a homomorphism $\pi^{*}: H_{G}^{*}\left(X_{G}^{s s} ; \mathbb{Q}\right) \rightarrow H_{T}^{*}\left(X_{G}^{s s} ; \mathbb{Q}\right)$. Because $T$ is a maximal torus, $\pi^{*}$ yields an isomorphism onto the submodule of elements invariant under the action of the Weyl group $W$ :

$$
\pi: H_{G}^{*}\left(X_{G}^{s s} ; \mathbb{Q}\right) \cong H_{T}^{*}\left(X_{G}^{s s} ; \mathbb{Q}\right)^{W}
$$

Date: September 7, 2013.

This work was supported by the NSF through a Graduate Research Fellowship. 
Moreover, there is a $W$-equivariant isomorphism

$$
H_{T}^{*}\left(X_{G}^{s s} ; \mathbb{Q}\right) \cong S \otimes_{S^{W}} H_{G}^{*}\left(X_{G}^{s s} ; \mathbb{Q}\right),
$$

where $S:=H_{T}^{*}(\operatorname{Spec} \mathbb{C} ; \mathbb{Q})$ is the $T$-equivariant cohomology of the point $\operatorname{Spec} \mathbb{C}$, and under this identification $\pi^{*}$ becomes $1 \otimes$ id. The $W$-anti-invariant elements $S^{a} \subseteq S$ form a free module of rank 1 over the subring $S^{W}$ of Weyl-invariant elements, and a generator is given by

$$
\Delta:=c_{\text {top }}(\mathfrak{g} / \mathfrak{b}),
$$

the top equivariant Chern class of the adjoint representation on $\mathfrak{g} / \mathfrak{b}$, where $\mathfrak{g}$ is the Lie algebra of $G$ and $\mathfrak{b}$ is the Lie algebra of a Borel subgroup containing $T$. Therefore, $\Delta \smile \pi^{*}(-)$ gives an isomorphism from $H_{G}^{*}\left(X_{G}^{s s} ; \mathbb{Q}\right)$ onto the submodule of $W$-anti-invariant elements of $H_{T}^{*}\left(X_{G}^{s s} ; \mathbb{Q}\right)$,

$$
\Delta \smile \pi^{*}(-): H_{G}^{*}\left(X_{G}^{s s} ; \mathbb{Q}\right) \stackrel{\cong}{\rightrightarrows} H_{T}^{*}\left(X_{G}^{s s} ; \mathbb{Q}\right)^{a} .
$$

The open inclusion $i: X_{G}^{s s} \hookrightarrow X_{T}^{s s}$ induces a homomorphism $i^{*}: H_{T}^{*}\left(X_{T}^{s s} ; \mathbb{Q}\right) \rightarrow H_{T}^{*}\left(X_{G}^{s s} ; \mathbb{Q}\right)$, and Brion's key observation is that $i^{*}$ is an isomorphism on the $W$-anti-invariant submodules:

$$
i^{*}: H_{T}^{*}\left(X_{T}^{s s} ; \mathbb{Q}\right)^{a} \cong H_{T}^{*}\left(X_{G}^{s s} ; \mathbb{Q}\right)^{a} .
$$

The composition $\phi:=\left(i^{*}\right)^{-1} \circ\left(\Delta \smile \pi^{*}\right)$ yields the desired isomorphism. Explicitly, if $\tilde{\sigma} \in$ $H_{T}^{*}\left(X_{T}^{s s} ; \mathbb{Q}\right)^{W}$ denotes some $W$-invariant lift of the class $\sigma \in H_{G}^{*}\left(X_{G}^{s s} ; \mathbb{Q}\right)$, that is if $i^{*} \tilde{\sigma}=\pi^{*} \sigma$, then $\phi$ can be described as

$$
\phi: \sigma \mapsto \Delta \smile \tilde{\sigma}
$$

The main goal. This paper addresses the question of how the isomorphism $\phi$ interacts with the integration pairings on the GIT quotients $X / / G$ and $X / / T$. When $X_{G}^{s s}=X_{G}^{s}$, there is a natural identification between the equivariant cohomology groups of the semi-stable locus and the ordinary cohomology groups of the GIT quotient,

$$
H_{G}^{*}\left(X_{G}^{s s} ; \mathbb{Q}\right) \cong H^{*}(X / / G ; \mathbb{Q}),
$$

(and similarly with $T$ in place of $G$ ). For any $\sigma_{1}, \sigma_{2} \in H^{*}(X / / G ; \mathbb{Q})$, one can then, in such a case, compare the integrals

$$
\int_{X / / G} \sigma_{1} \smile \sigma_{2} \stackrel{?}{\leftrightarrow} \int_{X / / T} \phi\left(\sigma_{1}\right) \smile \phi\left(\sigma_{2}\right) .
$$

Because $\phi\left(\sigma_{1}\right) \smile \phi\left(\sigma_{2}\right)=(\Delta \smile \Delta) \smile\left(\tilde{\sigma}_{1} \smile \tilde{\sigma}_{2}\right)$ and $i^{*}\left(\tilde{\sigma}_{1} \smile \tilde{\sigma}_{2}\right)=\pi^{*}\left(\sigma_{1} \smile \sigma_{2}\right)$, we may simplify the expression by defining $\sigma:=\sigma_{1} \smile \sigma_{2}$. Moreover, we consider the class $c_{\text {top }}(\mathfrak{g} / \mathfrak{t})$, where $\mathfrak{t} \subseteq \mathfrak{g}$ is the inclusion of the Lie algebra of $T$ in the adjoint representation on the Lie algebra of $G$, instead of the class $\Delta \smile \Delta$, which just differs from the former by the sign $(-1)^{\operatorname{dim} \mathfrak{g} / \mathfrak{b}}$. After these substitutions, the question becomes the comparison of the integrals $\int_{X / / G} \sigma$ and $\int_{X / / T} c_{\text {top }}(\mathfrak{g} / \mathfrak{t}) \smile \tilde{\sigma}$ for $\sigma \in H^{*}(X / / G ; \mathbb{Q})$.

Within an unpublished manuscript, Martin [24] provided an answer to the symplecto-geometric analogue of this question. There he proved the following formula for Hamiltonian actions of connected compact Lie groups $G$ on symplectic manifolds $X$ for which the moment map is proper and has 0 as a regular value:

$$
\int_{X / / G} \sigma=\frac{1}{|W|} \int_{X / / T} c_{\mathrm{top}}(\mathfrak{g} / \mathfrak{t}) \smile \tilde{\sigma},
$$

with $X / / G$ and $X / / T$ here denoting the symplectic reductions. Technically (0.1) only applies when $G$ and $T$ act freely on $\mu_{G}^{-1}(0)$ and $\mu_{T}^{-1}(0)$ respectively, but Martin proves a version of the formula 
that applies when the stabilizer groups of these actions are finite; this version involves a multiplicative factor equal to the ratio of the orders of the generic stabilizers (cf. [24, Thm. $\left.\mathrm{B}^{\prime}\right]$ ).

Martin's formula may be deduced from the Jeffrey-Kirwan-Witten non-abelian localization theorem [18, 32], although the proof Martin gave is much simpler than the proof of the general theorem [18. Thm. 8.1]. In addition to Martin's work [24], there has been a large body of literature devoted to understanding non-abelian localization; alternative approaches to the theorem may be found in the works of Guillemin and Kalkman [13], Paradan [25], and Vergne [30]. We note that the methods used in these works are analytic, and hence bound to characteristic 0 , while the methods used in the works of Brion, Ellingsrud-Strømme, and Brion-Joshua referenced above are algebraic.

New results. This article generalizes Martin's result to the algebraic setting of varieties $X$ over an arbitrary field $k$. Let $G$ be a reductive group over $k$ with a maximal torus $T \subseteq G$, and let $X$ be a projective (and possibly singular) $G$-variety with a $G$-linearized ample line bundle $\mathscr{L}$ for which $X_{T}^{s}=X_{T}^{s s} \neq \emptyset$. For any Chow 0 -cycle $\sigma \in A_{0}(X / / \mathbb{G})_{\mathbb{Q}}$ with non-zero degree, we are led to define the GIT integration ratio,

$$
r_{G, T}^{X, \sigma}:=\frac{\int_{X / / T} c_{\text {top }}(\mathfrak{g} / \mathfrak{t}) \frown \tilde{\sigma}}{\int_{X / / G} \sigma} \in \mathbb{Q},
$$

where the Chow class $\tilde{\sigma} \in A_{*}(X / / T)_{\mathbb{Q}}$ denotes an arbitrary lift of the class $\sigma$ (see Defn. 1.2.1).

Understanding the properties of the GIT integration ratio will guide us to the correct generalization of Martin's theorem. Since Definition 1.2.1 involves the pull-back of Chow classes by the morphism of Deligne-Mumford stacks $\left[X_{G}^{s s} / T\right] \rightarrow\left[X_{G}^{s s} / G\right]$, information about the stabilizer groups of the $G$ and $T$ actions is already encoded in our construction, and we shall not require a separate statement in the presence of non-trivial stabilizers.

Remark 0.2. We may immediately reduce our discussion to the case of a connected reductive group $G$ because $X_{G}^{s s}=X_{G_{0}}^{s s}$, where $G_{0} \subseteq G$ is the connected component of the identity (cf. [12, Prop. 1.15]), from which it follows quickly that

$$
r_{G, T}^{X, \sigma}=\left[G: G_{0}\right] \cdot r_{G_{0}, T}^{X, \pi^{*} \sigma},
$$

since the morphism $\pi:\left[X_{G_{0}}^{s s} / G_{0}\right] \rightarrow\left[X_{G}^{s s} / G\right]$ induced by the quotient $X_{G_{0}}^{s s} \rightarrow\left[X_{G}^{s s} / G\right]$ is finite and flat of degree $\left[G: G_{0}\right]$.

From now on, we assume that $G$ is a connected reductive group and $W$ is the associated Weyl group. Our first main result (proved in 93 ) concerns the invariance properties of the GIT integration ratio $r_{G, T}^{X, \sigma}$, which a priori could depend on the choice of lift $\tilde{\sigma}$ or the variety $X$. In fact, neither is the case:

Theorem 0.3. Let $G$ be a connected reductive group over an arbitrary field and $T \subseteq G$ a maximal torus. If $X$ is a projective $G$-variety with a G-linearized ample line bundle $\mathscr{L}$ for which $X_{T}^{s s}=X_{T}^{s}$ and $\sigma \in A_{0}(X / / G)$ is a Chow 0 -cycle satisfying $\int_{X / / G} \sigma \neq 0$, then the GIT integration ratio $r_{G, T}^{X, \sigma}$ defined as above depends not on the choice of $\sigma, T, \mathscr{L}$, or $X$. That is, $r_{G}:=r_{G, T}^{X, \sigma}$ is an invariant of the group $G$.

Connected reductive groups decompose, up to central isogeny, as the direct product of a torus and simple algebraic groups (cf. [2, §14]), and the GIT integration ratio can be computed in terms of the GIT integration ratios of the simple algebraic groups appearing in this decomposition. This is the content of our second main result (proved in $\$ 4$ ): 
Theorem 0.4. If $G$ is a connected reductive group over a field $k$ and there exists a central isogeny, $G_{1} \times \cdots \times G_{n} \times S \rightarrow G$, for connected reductive groups $G_{i}$ and a torus $S$, then

$$
r_{G}=\prod_{i=1}^{n} r_{G_{i}} .
$$

As a result of these theorems, the determination of the value $r_{G}$ for connected reductive groups $G$ is reduced to the computation of $r_{G}$ on a single example for each simple group. We do this explicitly in $\$ 5$ for the simple group $G=P G L(n)$, where we verify $r_{G}=n !=|W|$. Thus, we obtain a strictly algebraic proof of the following corollary:

Corollary 0.5. Let $G$ be a connected reductive group over a field $k$ and $T \subseteq G$ a maximal torus. If the root system of $G$ decomposes into irreducible root systems of type $\mathbf{A}_{n}$, for various $n \in \mathbb{N}$, then for any $G$-linearized ample line bundle on a projective $G$-variety $X$ over $k$ satisfying $X_{T}^{s}=X_{T}^{s s}$ and any Chow class $\sigma \in A_{0}(X / / G)_{\mathbb{Q}}$ with lift $\tilde{\sigma} \in A_{*}(X / / T)_{\mathbb{Q}}$,

$$
\int_{X / / G} \sigma=\frac{1}{|W|} \int_{X / / T} c_{\text {top }}(\mathfrak{g} / \mathfrak{t}) \frown \tilde{\sigma} .
$$

We can do even better than Corollary 0.5 if we do not restrict ourselves to purely algebraic arguments. Indeed, we may remove the restriction on root systems, proving that the GIT integration ratio is equal to the order of the Weyl group for any connected reductive group:

Amplification 0.6. Let $G$ be a connected reductive group over a field $k$ and $T \subseteq G$ a maximal torus. For any $G$-linearized ample line bundle on a projective $G$-variety $X$ over $k$ satisfying $X_{T}^{s}=X_{T}^{s s}$ and any Chow class $\sigma \in A_{0}(X / / G)_{\mathbb{Q}}$ with lift $\tilde{\sigma} \in A_{*}(X / / T)_{\mathbb{Q}}$,

$$
\int_{X / / G} \sigma=\frac{1}{|W|} \int_{X / / T} c_{\text {top }}(\mathfrak{g} / \mathfrak{t}) \frown \tilde{\sigma}
$$

The proof of this result uses the theories of relative GIT (cf. [27]) and specialization (cf. [11, $\S 20.3]$ ), along with Theorem 0.3 , to reduce the proof to the case of connected reductive groups over the complex numbers, where Martin's result [24, Thm. B'] applies. This argument is found near the end of the paper in 6

Acknowledgments. It is with pleasure that I thank my thesis advisor Johan de Jong, both for teaching me algebraic geometry and for his generous guidance that led me through the discovery of these results. I also thank Burt Totaro and the anonymous referees for their diligence in reading earlier manuscripts and for their valuable suggestions.

\section{NOTATION}

Reductive groups

- $k$ denotes a field and $\bar{k}$ an algebraic closure of $k$.

- $e \in T \subseteq B \subseteq G$ denotes a smooth, connected reductive group $G$ over $k$ with identity element $e$, a maximal torus $T$, and a Borel subgroup $B$.

- $\mathfrak{g}, \mathfrak{b}$, and $\mathfrak{t}$ denote respectively the Lie algebras of $G, B$, and $T$.

- $N_{T} \subseteq G$ denotes the normalizer of $T$ in $G$.

- $W:=T \backslash N_{T}$ denotes the Weyl group of right cosets of $T$ in its normalizer $N_{T}$.

- $\Phi$ denotes the root system corresponding to $G_{\bar{k}}$ and the maximal torus $T_{\bar{k}}$.

- $\Lambda^{*}(T)$ denotes the character group of $T$ and $\Lambda_{*}(T)$ the group of 1-parameter subgroups.

- $\bar{G}$ denotes a group that is the quotient of $G$ by some normal subgroup. 
Group actions and quotients

- $V$ denotes a finite dimensional $G$-representation over $k$.

- $\mathbb{P}(V)$ denotes the projective space of hyperplanes in $V$, so that $\Gamma(\mathbb{P}(V), \mathcal{O}(1))=V$.

- $X$ denotes a projective variety over $k$ with a right $G$-action and an ample $G$-linearized line bundle $\mathscr{L}$.

- $X_{G}^{s}, X_{G}^{s s}, X_{G}^{s s s}$ and $X_{G}^{u n}$ denote the loci of stable, semi-stable, strictly semi-stable, and unstable points for a specified $G$-linearized ample line bundle on a projective $G$-variety $X$.

- $X / / G$ the uniform categorical quotient of the semi-stable locus $X_{G}^{s s}$ by right $G$-action.

- $Y \times_{H} G$ denotes, for an algebraic space $Y$ with a right $G$-action and a closed subgroup $H \subseteq G$, the algebraic space comprising the quotient of the product $Y \times G$ by the settheoretically free $H$-action $(y, g) \cdot h:=\left(y \cdot h, h^{-1} \cdot g\right)$. If $Y$ is a scheme, then $Y \times_{H} G$ is often a scheme as well (cf. [7, Prop. 23]).

- $[Y / G]$ denotes the stack-theoretic quotient of a right $G$-variety $Y$ by the $G$-action.

- $B T:=[\operatorname{Spec} k / T]$ denotes the Artin stack that is the algebraic classifying space of $T$.

Chow theory

- $A_{*}(-)_{R}$ (resp. $A^{*}(-)_{R}$ ) denotes the Chow group (resp. operational Chow group), graded by dimension (resp. codimension), and with coefficients in $R$, an abelian group. Coefficients will be taken in $\mathbb{Z}$ by default when the group $R$ is omitted from notation.

- $A_{*}^{G}(-)_{R}$ (resp. $A_{G}^{*}(-)_{R}$ ) denotes the $G$-equivariant Chow group (resp. $G$-equivariant operational Chow group) with coefficients in an abelian group $R$.

- $\int_{Y} \sigma \in R$ denotes the degree of a Chow class $\sigma \in A_{0}(Y)_{R}$ on a proper variety $Y$ over $k$, computed via proper push-forward by the structure morphism.

$\cdot \Delta:=c_{\text {top }}(\mathfrak{g} / \mathfrak{b}) \in A^{*}(B T)$ and $c_{\text {top }}(\mathfrak{g} / \mathfrak{t}) \in A^{*}(B T)$ are the top Chern classes of the universal $T$-equivariant vector bundles induced by the adjoint representations.

\section{LIFTING CLASSES ON SMOOTH VARIETIES}

The goal of this section is to prove if $X$ is a smooth $G$-variety over an arbitrary field $k$ with a $G$-linearized ample line bundle for which $X_{T}^{s s}=X_{T}^{s}$, then any Chow class $\sigma \in A_{*}(X / / G)_{\mathbb{Q}}$ gives rise to a well-defined class $\Delta \frown \tilde{\sigma} \in A_{*}(X / / T)_{\mathbb{Q}}$ that is independent of the choice of lift $\tilde{\sigma}$, as defined in 1.2 As a consequence, the independence of the Chow class $c_{\text {top }}(\mathfrak{g} / \mathfrak{t}) \frown \tilde{\sigma}$ follows immediately since

$$
c_{\text {top }}(\mathfrak{g} / \mathfrak{t})=(-1)^{|\Phi| / 2} \Delta \frown \Delta .
$$

It is this independence that is needed to make the GIT integration ratio $r_{G, T}^{X, \sigma}$ well-defined. The case of singular $X$ is treated separately in $\S_{2}$.

1.1. Geometric invariant theory. We begin by briefly reviewing geometric invariant theory, mainly to set our conventions.

Definition 1.1.1. Let $X$ be a projective $G$-variety over $k$ and $\mathscr{L}$ a $G$-linearized ample line bundle on $X$, that is, an ample line bundle $\pi: \mathscr{L} \rightarrow X$ along with $G$-actions on $\mathscr{L}$ and $X$ for which $\pi$ is equivariant and on whose fibres $G$ acts linearly.

- The semi-stable locus is the open subscheme defined by

$$
X_{G}^{s s}:=\left\{x \in X: \exists n>0 \text { and some } \phi \in \Gamma\left(X, \mathscr{L}^{\otimes n}\right)^{G} \text { satisfying } \phi(x) \neq 0\right\} .
$$


- The stable1 locus is the open subscheme defined by

$$
X_{G}^{s}:=\left\{x \in X_{G}^{s s}: x \cdot G \subseteq X_{G}^{s s} \text { is a closed subscheme and }\left|\operatorname{stab}_{G} x\right|<\infty\right\} .
$$

- The unstable locus is defined to be $X_{G}^{u n}:=X \backslash X_{G}^{s s}$.

- The strictly semi-stable locus is defined to be $X_{G}^{s s s}:=X_{G}^{s s} \backslash X_{G}^{s}$.

The following theorem justifies the making of the above definitions:

Theorem 1.1.2 (Mumford). The semi-stable locus for a $G$-linearized line bundle $\mathscr{L}$ on a projective $G$-variety $X$ admits a uniform categorical quotient $\pi: X_{G}^{s s} \rightarrow X / / G$ called the GIT quotient of $X$ by $G$. Moreover, some positive tensor power of $\mathscr{L}$ descends to an ample line bundle on the projective variety $X / / G$, and the restriction of $\pi$ to the stable locus is a geometric quotient.

Proof. See [12, Thm. 1.10].

Next we describe how to compute the stable and semi-stable loci in practice. As these loci are compatible with field extensions (cf. [12, Prop. 1.14]), we may assume that we are working over the algebraically closed field $\bar{k}$. Let $T \subseteq G$ be a maximal torus with character group $\Lambda^{*}(T)$. Equivariantly embed $X$ into $\mathbb{P}(V)$ for some $G$-representation $V$ so that $\mathcal{O}_{\mathbb{P}(V)}(1)$ pulls-back to the induced $G$-linearized line bundle $\mathscr{L}^{\otimes n}$, for some $n>0$. We will call such an embedding compatible. The $G$-representation structure on $V$ endows $\mathbb{P}(V)$ with a $G$-action and a $G$-linearization on $\mathcal{O}(1)$ for which both $\mathbb{P}(V)_{G}^{s s} \cap X=X_{G}^{s s}$ and $\mathbb{P}(V)_{G}^{s} \cap X=X_{G}^{s}$. Since $\bar{k}$ is algebraically closed, $T$ is diagonalizable and hence $V$ decomposes as the direct sum of weight spaces $V=\oplus_{\chi \in \Lambda^{*}(T)} V_{\chi}$.

Definition 1.1.3. For any $x \in X \subseteq \mathbb{P}(V)$ as above, the state of $x$ is defined to be

$$
\Xi(x):=\left\{\chi \in \Lambda^{*}(T): \exists v \in V_{\chi} \text { such that } v(x) \neq 0\right\} .
$$

We now state in the above notation the following well-known numerical criterion for stability:

Theorem 1.1.4 (Hilbert-Mumford criterion). A point $x \in X$ is T-semi-stable for the induced $T$ linearization on $\mathscr{L}$ if and only if 0 is in the convex hull of $\Xi(x)$ in $\Lambda^{*}(T) \otimes \mathbb{Q}$. A point $x \in X$ is $T$-stable if and only if 0 is in the interior of the convex hull of $\Xi(x)$. Furthermore,

$$
X_{G}^{s s}=\bigcap_{g \in G} X_{T}^{s s} \cdot g, \text { and } X_{G}^{s}=\bigcap_{g \in G} X_{T}^{s} \cdot g .
$$

Proof. See [12, Thm. 2.1].

Remark 1.1.5. A corollary of the Hilbert-Mumford criterion is that the condition in T-stability that all semi-stable points are stable, $X_{T}^{s s}=X_{T}^{s}$, automatically implies the analogous condition in $G$-stability, $X_{G}^{s s}=X_{G}^{s}$.

We conclude this review by presenting a stratification that describes the structure of the unstable locus. The stratification is due to Kirwan, but relies on the previous works of Hesselink [15, 16], Kempf [20] and Ness [21].

Theorem 1.1.6 (Kirwan). Let $X$ be a projective $G$-variety, along with a $G$-linearized ample line bundle $\mathscr{L}$, over an algebraically closed field $\bar{k}$. The unstable locus $X_{G}^{u n}$ admits a G-equivariant stratification,

$$
X_{G}^{u n}=\bigcup_{\beta \in \mathbf{B}} S_{\beta},
$$

indexed by a finite partially ordered set $\mathbf{B}$, with the following properties:

\footnotetext{
${ }^{1}$ Sometimes in the literature this is referred to as "properly stable".
} 
(1) $S_{\beta} \subseteq X_{G}^{u n}$ is a locally closed G-equivariant subscheme.

(2) $S_{\beta} \cap S_{\beta^{\prime}}=\emptyset$ for $\beta \neq \beta^{\prime}$.

(3) $\overline{S_{\beta}} \subseteq \bigcup_{\beta^{\prime} \geq \beta} S_{\beta^{\prime}}$.

(4) There exist parabolic subgroups $T \subseteq P_{\beta} \subseteq G$, locally closed $P_{\beta}$-closed subschemes $Y_{\beta} \subseteq$ $S_{\beta} \cap X_{T}^{u n}$, and a surjective G-equivariant morphism,

$$
\phi: Y_{\beta} \times_{P_{\beta}} G \rightarrow S_{\beta},
$$

induced by the multiplication morphism $Y_{\beta} \times G \rightarrow S_{\beta}$.

If moreover $X$ is smooth, then there exists a surjection of reductive groups $\pi: G \rightarrow \bar{G}$ such that the $G$-actions on $X$ and $\mathscr{L}$ are induced by $\bar{G}$-actions, and $\phi$ descends to an isomorphism $\bar{\phi}: Y_{\beta} \times_{\bar{P}_{\beta}} \bar{G} \rightarrow S_{\beta}$, where $\bar{P}_{\beta}=\pi\left(P_{\beta}\right)$. If char $k=0$ or $X$ compatibly embeds into $\mathbb{P}(V)$ for some faithful $G$-representation $V$, then one can take $G=\bar{G}$.

Proof. If $X$ compatibly embeds into $\mathbb{P}(V)$ for some faithful $G$-representation $V$ then the theorem holds, with $\phi$ an isomorphism when $X$ is smooth, by [22, $\S 12-\S 13]$. The general result can then be reduced to this case: let $\bar{G}$ be the image of the homomorphism $h: G \rightarrow G L(V)$ and let $\bar{P}_{\beta}$ be the parabolic subgroups determined by this faithful $\bar{G}$ action as in [22, $\S 12-\S 13]$; the theorem then holds with $P_{\beta}:=h^{-1}\left(\bar{P}_{\beta}\right)^{\text {red }}$. We remark that unlike $\bar{\phi}$, the morphism $\phi$ may not be an isomorphism for general smooth $X$ because $h^{-1}\left(\bar{P}_{\beta}\right)$ may be non-reduced if the characteristic is positive.

1.2. Lifts. We now define precisely what it means to lift a Chow class $\sigma \in A_{*}(X / / G)_{\mathbb{Q}}$ to a class $\tilde{\sigma} \in A_{*}(X / / T)_{\mathbb{Q}}$. We make use of the notion of Chow groups of quotient stacks, a review of which may be found in the appendix to this paper. The main result we will need is that when $X_{G}^{s s}=X_{G}^{s}$, the quotient $\left[X_{G}^{s s} / G\right]$ is a proper Deligne-Mumford stack with a morphism $\phi^{G}:\left[X_{G}^{s s} / G\right] \rightarrow X / / G$ that induces an isomorphism of rational Chow groups (cf. Thm. A.1.1):

$$
\phi_{*}^{G}: A_{*}\left(\left[X_{G}^{s s} / G\right]\right)_{\mathbb{Q}} \cong A_{*}(X / / G)_{\mathbb{Q}} .
$$

Via the identification $\phi_{*}^{G}$, we may think of a Chow class $\sigma \in A_{*}\left(\left[X_{G}^{s s} / G\right]\right)_{\mathbb{Q}}$ equivalently as $\phi_{*}^{G}(\sigma) \in A_{*}(X / / G)_{\mathbb{Q}}$, and we will henceforth denote both classes by the symbol $\sigma$.

Definition 1.2.1. If $X$ is a projective $G$-variety with a $G$-linearized ample line bundle for which $X_{T}^{s s}=X_{T}^{s}$, then a lift of $\sigma \in A_{d}(X / / G)_{\mathbb{Q}}$ is a class $\tilde{\sigma} \in A_{d+\operatorname{dim}(G / T)}(X / / T)_{\mathbb{Q}}$ that satisfies $i^{*}(\tilde{\sigma})=f^{*}(\sigma)$, where $i:\left[X_{G}^{s s} / T\right] \hookrightarrow\left[X_{T}^{s s} / T\right]$ is the open immersion, and $f:\left[X_{G}^{s s} / T\right] \rightarrow$ $\left[X_{G}^{s s} / G\right]$ is the flat fibration with fibre $G / T$.

Remark 1.2.2. By the right exact sequence of Chow groups

$$
\left.A_{*}\left(\left[X_{G}^{u n} \cap X_{T}^{s s} / T\right]\right)_{\mathbb{Q}} \rightarrow A_{*}\left(\left[X_{T}^{s s} / T\right]\right)_{\mathbb{Q}} \stackrel{i^{*}}{\rightarrow} A_{*}\left(X_{G}^{s s} / T\right]\right)_{\mathbb{Q}} \rightarrow 0,
$$

any two lifts of a Chow class differ by the push-forward of an element of $A_{*}\left(\left[X_{G}^{u n} \cap X_{T}^{s s} / T\right]\right)_{\mathbb{Q}}$.

1.3. Vanishing on a stratum. Throughout this subsection, we assume that $X$ is a smooth, projective $G$-variety with a $G$-linearized ample line bundle over an algebraically closed field $\bar{k}$. In light of Remark 1.2.2 to show that $\Delta \frown \tilde{\sigma}$ is independent of the choice of lift, it suffices to show that $\Delta$ kills all elements in the image of $A_{*}\left(\left[X_{G}^{u n} \cap X_{T}^{s s} / T\right]\right)_{\mathbb{Q}}$. For now we just prove that $\Delta$ vanishes on the $T$-semi-stable locus of each stratum, $S_{\beta} \cap X_{T}^{s s}$. Since $X$ is smooth, Theorem 1.1.6 guarantees that the stratum $S_{\beta}$ is fibred over a flag variety $\bar{P}_{\beta} \backslash \bar{G}$ of right $\bar{P}_{\beta}$-cosets, and so we begin our study here.

We require some notation related to the Weyl group $W:=T \backslash N_{T}$. For a parabolic subgroup $P \subseteq G$ containing the maximal torus $T$, denote by $W_{P}$ the subgroup $T \backslash\left(N_{T} \cap P\right) \subseteq W$. Let the 
symbol $\dot{w}$ denote a choice of representative in $N_{T}$ of a Weyl class $w \in W$, and let the symbol $\bar{w}$ denote the image of $w$ in $W_{P} \backslash W$.

Lemma 1.3.1. Let $P \subseteq G$ be a parabolic subgroup containing $T$, and denote by $\mathfrak{p}$ its Lie algebra. The inclusion $i: W_{P} \backslash W \rightarrow P \backslash G$, defined by $\bar{w} \mapsto P \dot{w}$, is the inclusion of the T-fixed points of $P \backslash G$, and the Gysin pull-back of the T-equivariant class $[P] \in A_{*}^{T}(P \backslash G)$ is given by

$$
i^{*}([P])=c_{\text {top }}(\mathfrak{g} / \mathfrak{p}) \cdot[\bar{e}] \in A_{*}^{T}\left(W_{P} \backslash W\right),
$$

where the $T$-action on $\mathfrak{g} / \mathfrak{p}$ is via the adjoint representation.

Proof. It is well-known that the $T$-invariant points of $P \backslash G$ are precisely $W_{P} \backslash W$. The Chow group $A_{*}^{T}\left(W_{P} \backslash W\right)$ is a free $A^{*}(B T)$-module with basis given by the elements of $W_{P} \backslash W$. The element $P \in P \backslash G$ is an isolated, nonsingular fixed point, hence $i^{*}([P])$ equals the product of $[\bar{e}]$ with the $T$-equivariant top Chern class of the normal bundle of $P$ at $P$, which is just $c_{\text {top }}(\mathfrak{g} / \mathfrak{p})$.

Lemma 1.3.2. Let $P \subseteq G$ be a parabolic subgroup containing $T$, and let $U \subseteq P \backslash G$ denote the open complement of the $T$-fixed points $W_{P} \backslash W \hookrightarrow P \backslash G$. As an element of the T-equivariant operational Chow group of $U$,

$$
\Delta=0 \in A_{T}^{*}(U) .
$$

Proof. The variety $U$ is smooth, so by Poincaré duality (Theorem A.2.4) it suffices to prove that $\Delta \frown[U]=0 \in A_{*}^{T}(U)$. Let $X:=P \backslash G$ denote the flag variety, and let $i: X^{T}=W_{P} \backslash W \rightarrow X$ denote the inclusion of the $T$-fixed points. By the right-exact sequence of Chow groups

$$
A_{*}^{T}\left(X^{T}\right) \stackrel{i_{*}}{\rightarrow} A_{*}^{T}(X) \rightarrow A_{*}^{T}(U) \rightarrow 0,
$$

it suffices to show that $\Delta \frown[X]$ is in the image of $i_{*}$. Since $X$ is a smooth, projective variety, by the localization theorem (Theorem A.1.3), $i^{*}: A_{T}^{*}(X) \rightarrow A_{T}^{*}\left(X^{T}\right)$ is an injective morphism of $A^{*}(B T)$-algebras. Thus, it suffices to prove that $i^{*}(\Delta \frown[X])$ is in the image of $i^{*} \circ i_{*}$.

The ring $A^{*}\left(X^{T}\right)$ is a free $A^{*}(B T)$-module with basis given by $\left\{[\bar{w}]: \bar{w} \in W_{P} \backslash W\right\}$. In terms of this basis,

$$
i^{*}(\Delta \frown[X])=\sum_{\bar{w} \in W_{P} \backslash W} \Delta \cdot[\bar{w}] .
$$

By Lemma 1.3.1, $i^{*} \circ i_{*}([\bar{e}])=c_{\text {top }}(\mathfrak{g} / \mathfrak{p}) \cdot[\bar{e}]$. Since $i$ is a $W$-equivariant inclusion, $i^{*} \circ i_{*}$ is compatible with the $W$-action. Therefore for any $w \in W$,

$$
i^{*} \circ i_{*}([\bar{w}])=\left(\prod_{\alpha \in \Phi(\mathfrak{g} / \mathfrak{p})} \alpha w\right) \cdot[\bar{w}],
$$

where $\Phi(\mathfrak{g} / \mathfrak{p}) \subseteq \Phi$ denotes the subset of roots that appear in the diagonalization of the adjoint action on $\mathfrak{g} / \mathfrak{p}$. Notice that $\Phi(\mathfrak{g} / \mathfrak{p})$ is a subset of the negative roots $\Phi^{-}:=\Phi(\mathfrak{g} / \mathfrak{b})$, for any choice of Borel subgroup $B \subseteq P$. Hence, for the Chow class $\beta_{w}:=\prod_{\alpha \in \Phi^{-} \backslash \Phi(\mathfrak{g} / \mathfrak{p})} \alpha w \in A^{*}(B T)$,

$$
i^{*} \circ i_{*}\left(\beta_{w} \cdot[\bar{w}]\right)=\left(\prod_{\alpha \in \Phi^{-}} \alpha w\right) \cdot[\bar{w}] .
$$

Since $\Delta=\prod_{\alpha \in \Phi^{-}} \alpha$, it follows that $\prod_{\alpha \in \Phi^{-}} \alpha w=\operatorname{det}(w) \cdot \Delta$. Therefore, $\Delta \frown[X]$ is in the image of $i^{*} \circ i_{*}$ :

$$
\sum_{\bar{w} \in W_{P} \backslash W} \Delta \cdot[\bar{w}]=i^{*} \circ i_{*}\left(\sum \operatorname{det}(w) \cdot \beta_{w} \cdot[\bar{w}]\right) .
$$


The previous two lemmas are directly analogous to results in equivariant cohomology over $\mathbb{C}$ due to Brion (cf. [3, Lem. 2]). The stratification of Theorem 1.1.6 is more subtle for fields of characteristic $p$, and we hence must stray from Brion's approach. Even over $\mathbb{C}$, our method has the added benefit that most results hold with integer, and not just rational, coefficients.

Lemma 1.3.3. Let $\pi: G \rightarrow \bar{G}$ be a surjection of reductive groups over an algebraically closed field $\bar{k}$. If $\bar{k}$ is characteristic 0 let $p=1$ and otherwise let $p:=$ char $k$. If $f: Y \rightarrow U \subseteq \bar{P} \backslash \bar{G}$ is an equivariant morphism of $\bar{G}$-varieties, where $\bar{P}:=\pi(P)$ and $U$ is the complement of the $T$-fixed points, then

$$
\Delta=c_{\text {top }}(\mathfrak{g} / \mathfrak{b})=0 \in A_{T}^{*}(Y)_{\mathbb{Z}\left[\frac{1}{p}\right]} .
$$

Proof. Lemma 1.3.2 immediately implies that the class $\bar{\Delta}:=c_{\text {top }}(\overline{\mathfrak{g}} / \overline{\mathfrak{b}})=0 \in A_{\bar{T}}^{*}(Y)_{\mathbb{Z}}$, for the Lie algebras $\overline{\mathfrak{g}}$ and $\overline{\mathfrak{b}}$ of $\bar{G}$ and the Borel subgroup $\bar{B}=\pi(B)$, respectively. Therefore, it suffices to show that the pull-back of $\bar{\Delta}$ divides $p^{N} \cdot \Delta$ in the ring $A_{T}^{*}(Y)$ for some $N \gg 0$. This follows from [28, 5.1-2], which states that $\pi^{*}: \Lambda^{*}(\bar{T}) \rightarrow \Lambda^{*}(T)$ is an injective homomorphism that takes the roots of $\bar{G}$ into the set $\bigcup_{n=0}^{\infty}\left(p^{n} \cdot \Phi\right) \subseteq \Lambda^{*}(T)$.

From the previous lemma, we derive that $\Delta$ is zero on the $T$-semi-stable locus of each stratum $S_{\beta} \cap X_{T}^{s s}$.

Lemma 1.3.4. Let $X$ be a smooth, projective $G$-variety with a $G$-linearized ample line bundle over $\bar{k}$. As an element of the T-equivariant operational Chow group of $S_{\beta} \cap X_{T}^{s s}$,

$$
\Delta=0 \in A_{T}^{*}\left(S_{\beta} \cap X_{T}^{s s}\right)_{\mathbb{Z}\left[\frac{1}{p}\right]},
$$

where $p>0$ is equal to the prime characteristic unless the characteristic is 0 , in which case $p=1$.

Proof. By Theorem 1.1.6 there is a $G$-equivariant morphism $\pi: S_{\beta} \rightarrow \bar{P}_{\beta} \backslash \bar{G}$ with $\pi^{-1}\left(\bar{P}_{\beta}\right)=$ $Y_{\beta} \subseteq X_{T}^{u n}$. The normalizer $N_{T}$ preserves the unstable locus $X_{T}^{u n}$, so in particular $Y_{\beta} \cdot N_{T} \subseteq X_{T}^{u n}$. Hence, the image of $\pi$ restricted to $S_{\beta} \cap X_{T}^{s s}$ is contained in $U$, the open complement in $\bar{P}_{\beta} \backslash \bar{G}$ of the $T$-fixed points $\bar{P}_{\beta} \backslash \bar{P}_{\beta} \cdot N_{T}$. We conclude by invoking Lemma 1.3.3.

1.4. Unions of strata. We continue to assume that $X$ is smooth over an algebraically closed field $\bar{k}$, and we extend the vanishing of $\Delta$ on a stratum to the vanishing over the entire locus $X_{G}^{u n} \cap X_{T}^{s s}$.

Proposition 1.4.1. Let $X$ be a smooth, projective $G$-variety with a $G$-linearized ample line bundle over $\bar{k}$. The operational Chow class $\Delta$ annihilates every class in $A_{*}^{T}\left(X_{G}^{u n} \cap X_{T}^{s s}\right)_{\mathbb{Z}\left[\frac{1}{p}\right]}$, where $p>0$ is equal to the prime characteristic unless the characteristic is 0 , in which case $p=1$.

Proof. Label $\mathbf{B}=\left\{\beta_{1}, \ldots, \beta_{n}\right\}$ so that $\mathcal{S}_{k}:=\bigcup_{i<k} S_{\beta_{i}}$ is a closed subvariety of $X_{G}^{u n}$, for each $1 \leq$ $k \leq n$; this is possible by Theorem 1.1.6 (3). We proceed to inductively prove that $\Delta$ annihilates every class in $A_{*}^{T}\left(\mathcal{S}_{k} \cap X_{T}^{s s}\right)_{\mathbb{Z}}$ for all $k=1, \ldots, n$, with $k=n$ being the desired result. By Lemma 1.3.4 the assertion is true when $k=1$ and $\mathcal{S}_{1}=S_{\beta 1}$ is an individual stratum. Assume the result holds for $1 \leq k<n$. By Proposition A.1.2, it suffices to show that $\Delta \frown[Y]=0 \in$ $A_{*}^{T}\left(\mathcal{S}_{k+1} \cap X_{T}^{s s}\right)$ for any $T$-invariant subvariety $Y \hookrightarrow \mathcal{S}_{k+1} \cap X_{T}^{s s}$. Since $\mathcal{S}_{k} \hookrightarrow \mathcal{S}_{k+1}$ is a closed immersion, the inductive hypothesis will imply $\Delta \frown[Y]=0$ whenever $Y$ is completely contained in $\mathcal{S}_{k}$. As $\mathcal{S}_{k+1}=S_{\beta_{k+1}} \cup \mathcal{S}_{k}$, the only remaining case is when $Y$ intersects $S_{\beta_{k+1}}$ nontrivially.

For the sake of clarity, denote $\beta:=\beta_{k+1}$. Consider the birational map $\overline{S_{\beta}} \rightarrow \bar{P}_{\beta} \backslash \bar{G}$ defined on $S_{\beta}$ by $S_{\beta} \cong Y_{\beta} \times_{\bar{P}_{\beta}} \bar{G} \rightarrow \bar{P}_{\beta} \backslash \bar{G}$ as in Theorem 1.1.6. Our strategy will be to partially resolve the locus of indeterminacy in the following manner: 


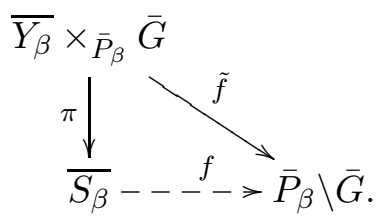

The morphism $\pi$ is proper, since $\bar{P}_{\beta} \backslash \bar{G}$ is so, and is an isomorphism when restricted to the dense open $S_{\beta} \subseteq \overline{S_{\beta}}$. Moreover, $\tilde{f}^{-1}\left(\bar{P}_{\beta}\right)=\overline{Y_{\beta}}$, and by $G$-invariance,

$$
\tilde{f}^{-1}\left(\bar{P}_{\beta} W\right)=\overline{Y_{\beta}} \times_{\bar{P}_{\beta}} \bar{P}_{\beta} \cdot W .
$$

Since $N_{T}$ preserves $X_{T}^{u n}$ and $\overline{Y_{\beta}} \subseteq X_{T}^{u n}$, it follows that $\pi\left(\tilde{f}^{-1}\left(P_{\beta} W\right)\right) \subseteq X_{T}^{u n}$, showing that the above diagram restricts to

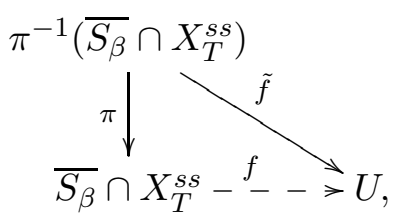

where $U \subseteq \bar{P}_{\beta} \backslash \bar{G}$ is the open complement of the locus of $T$-fixed points.

Let $\tilde{Y}$ denotes the strict transform of $Y$ under the birational morphism $\pi$. From Lemma 1.3.3 it follows that $\Delta \frown[\tilde{Y}]=0$. At the same time, $\pi_{*}(\Delta \frown[\tilde{Y}])=\Delta \frown[Y]$ by the projection formula, so $\Delta \frown[Y]=0 \in A_{*}^{T}\left(\overline{S_{\beta}} \cap X_{T}^{s S}\right)$. By the projection formula for the closed immersion $\overline{S_{\beta}} \hookrightarrow \mathcal{S}_{k+1}$, we conclude that $\Delta \frown[Y]=0 \in A_{*}^{T}\left(\mathcal{S}_{k+1} \cap X_{T}^{s s}\right)$.

1.5. Arbitrary fields. We now relax our previous assumption, henceforth allowing an arbitrary base field $k$. The stratification of Theorem 1.1.6 is constructed over an algebraically closed field, so our previous arguments do not immediately apply. However, if we weaken our statements by ignoring torsion, considering only Chow groups with rational coefficients, our previous results quickly extend to this more general setting. We outline the proof of the following well-established lemma (cf. [1, Lem. 1A.3]) for the reader's convenience.

Lemma 1.5.1. If $X$ is a variety over a field $k$, then any field extension $K / k$ induces an injective morphism between Chow groups with rational coefficients: $A_{*}(X)_{\mathbb{Q}} \hookrightarrow A_{*}\left(X_{K}\right)_{\mathbb{Q}}$.

Proof. If a field extension $E / k$ is the union of a directed system of sub-extensions $E_{i} / k$, then $A_{*}\left(X_{E}\right)=\lim A_{*}\left(X_{E_{i}}\right)$. We may apply this result to the extension $K / k$ to reduce the proof to the two cases: $\overrightarrow{K / k}$ is finite; or $K=k(x)$ for a transcendental element $x$. Let $\phi: X_{K} \rightarrow X$ denote the base change morphism.

If $K / k$ is finite, then $\phi$ is flat and proper, and the composition $\phi_{*} \circ \phi^{*}$ is simply multiplication by $[K: k]$. This is an isomorphism since coefficients are rational, and hence $\phi^{*}$ is injective.

If $K=k(x)$, then $X_{K}$ is the generic fibre of the projection $\pi: X \times \mathbb{P}_{k}^{1} \rightarrow \mathbb{P}_{k}^{1}$. If $\psi: X \times \mathbb{P}_{k}^{1} \rightarrow X$ denotes the other projection and $j: X_{K} \rightarrow X \times \mathbb{P}_{k}^{1}$ is the inclusion of the generic fibre, then $\phi=\psi \circ j$. There is an isomorphism of Chow groups,

$$
A_{i+1}\left(X \times \mathbb{P}_{k}^{1}\right)_{\mathbb{Q}} \cong A_{i}(X)_{\mathbb{Q}} \oplus A_{i+1}(X)_{\mathbb{Q}} \cdot t,
$$

where $t$ is the class associated to a fibre of $\pi$. The pull-back $\psi^{*}: A_{i}(X)_{\mathbb{Q}} \rightarrow A_{i+1}\left(X \times \mathbb{P}_{k}^{1}\right)_{\mathbb{Q}}$ is identified with id $\oplus 0: A_{i}(X)_{\mathbb{Q}} \rightarrow A_{i}(X)_{\mathbb{Q}} \oplus A_{i+1}(X)_{\mathbb{Q}} \cdot t$ (cf. [11, Thm. 3.3]). As schemes,

$$
X_{K}=\underset{\emptyset \neq U \subseteq \mathbb{P}^{1}}{\lim } X \times U
$$


and there is an induced isomorphism on the level of Chow groups:

$$
A_{i}\left(X_{K}\right)_{\mathbb{Q}} \cong \lim _{\emptyset \neq U \subseteq \mathbb{P}^{1}} A_{i+1}(X \times U)_{\mathbb{Q}} \cong A_{i}(X)_{\mathbb{Q}} \oplus 0
$$

Under this identification, the pull-back $j^{*}: A_{i+1}\left(X \times \mathbb{P}_{k}^{1}\right)_{\mathbb{Q}} \rightarrow A_{i}\left(X_{K}\right)_{\mathbb{Q}}$ is the projection $A_{i}(X)_{\mathbb{Q}} \oplus A_{i}(X)_{\mathbb{Q}} \cdot t \rightarrow A_{i}(X)_{\mathbb{Q}}$. From this description, it is clear that $\phi^{*}=j^{*} \circ \psi^{*}$ is an isomorphism, ergo injective.

Remark 1.5.2. When the extension $K / k$ is purely transcendental, the above proof actually shows that the pull-back $A_{*}(X) \hookrightarrow A_{*}\left(X_{K}\right)$ is an injective group homomorphism between Chow groups with integer coefficients.

Proposition 1.5.3. Let $X$ be a smooth, projective $G$-variety with a $G$-linearized ample line bundle over an arbitrary field $k$. The operational Chow class $\Delta$ annihilates every class in $A_{*}^{T}\left(X_{G}^{u n} \cap\right.$ $\left.X_{T}^{s s}\right)_{\mathbb{Q}}$

Proof. Lemma 1.5.1 reduces the proof to the case of an algebraically closed base field, which is the content of Proposition 1.4.1

We state an immediate corollary that we will later extend to the case of singular varieties (cf. Prop. 2.3.1).

Corollary 1.5.4. Let $X$ be a smooth, projective $G$-variety over a field $k$, with a $G$-linearized ample line bundle for which $X_{T}^{s s}=X_{T}^{s}$. If $\tilde{\sigma}_{0}, \tilde{\sigma}_{1} \in A_{*}(X / / T)_{\mathbb{Q}}$ are two lifts of the same class $\sigma \in$ $A_{*}(X / / G)_{\mathbb{Q}}$, then for any operational Chow class $c \in A^{*}(X / / T)_{\mathbb{Q}}$,

$$
\int_{X / / T} c \frown\left(\Delta \frown \tilde{\sigma}_{0}\right)=\int_{X / / T} c \frown\left(\Delta \frown \tilde{\sigma}_{1}\right)
$$

\section{SiNGULAR VARIETIES AND STRICTLY SEMI-STABLE POINTS}

We wish to extend the results of $₫ 1$ to the case of singular $X$ by studying a compatible closed immersion $j: X \hookrightarrow \mathbb{P}(V)$, but several obstacles must be overcome. The first of which is the regrettable fact that the push-forward map $j_{*}: A_{*}\left(\left[X_{T}^{s s} / T\right]\right)_{\mathbb{Q}} \rightarrow A_{*}\left(\left[\mathbb{P}(V)_{T}^{s s} / T\right]\right)_{\mathbb{Q}}$ is not injective in general, thus preventing an easy reduction to the smooth case. Suppose we attempt to circumvent this problem by limiting our ambitions to showing that any Chow class $\sigma \in A_{*}(X / / G)_{\mathbb{Q}}$ gives rise to a well-defined numerical equivalence class $\Delta \frown \tilde{\sigma}$ independent of the choice of lift $\tilde{\sigma}$. Ideally the closed immersion $j$ would induce an injective map between the groups of algebraic cycles modulo numerical equivalence, and we would reduce our proof to the smooth case. Unfortunately, there is the possibility that $\mathbb{P}(V)_{T}^{s s} \neq \mathbb{P}(V)_{T}^{s}$, and in this case there is no notion of numerical equivalence on the Artin stack $\left[(\mathbb{P}(V))_{T}^{s s} / T\right]$ (cf. [9]). Once again, the naïve argument breaks down.

Our solution is to build an auxiliary smooth, projective $G$-variety $Y$ with a $G$-linearized ample line bundle for which $Y_{T}^{s s}=Y_{T}^{s}$, and then to relate integration on $X / / G$ and $X / / T$ to integration on $Y / / G$ and $Y / / T$. If one is guaranteed $G$-equivariant resolutions of singularities (e.g. if char $k=$ 0 ), then a result of Reichstein [26] generalizes the partial desingularizations of Kirwan [23] and produces such an auxiliary variety $Y$. Since resolution of singularities is still an open problem in positive characteristic, we provide an independent construction. As an application of our method, we prove in $\$ 2.3$ an extension of Corollary 1.5 .4 to singular varieties. 
2.1. Construction. The auxiliary variety $Y$ will be defined as an iterated product of the flag variety of right $B$-cosets, for a Borel subgroup $B$ containing the maximal torus $T$. The only delicate point is the choice of a suitable $G$-linearized line bundle, which will rely upon an understanding of equivariant line bundles over flag varieties.

Lemma 2.1.1. For any weight $\chi \in \Lambda^{*}(T)$ in the interior of the positive Weyl chamber, the line bundle $\mathscr{L}(\chi):=\mathbb{A}^{1} \times_{\chi, B} G$ is a very ample $G$-linearized line bundle on $B \backslash G$. For any nontrivial subtorus $T^{\prime} \subseteq T$ stabilizing some point $B g \in B \backslash G$, the induced action on the fibre of $\mathscr{L}(\chi)$ over $B g$ is by the restriction to $T^{\prime}$ of the weight $(\chi \cdot w)$, where $w \in W$ corresponds to the Bruhat cell containing $B g$.

Proof. That $\mathscr{L}(\chi)$ is a very ample line bundle on $B \backslash G$ is simply a rephrasing of the standard construction in representation theory of highest weight modules for dominant weights. The Bruhat decomposition states that $B \backslash G=\coprod_{w \in W} B w U$, for $U \subseteq B$ the maximal normal unipotent subgroup. Furthermore, denoting by $\dot{w} \in N_{T}$ a lift of the element $w \in W=T \backslash N_{T}$, there is an isomorphism

$$
\phi: B \times U_{w} \rightarrow B \dot{w} U \subseteq G
$$

sending $(b, u) \mapsto b \dot{w} u$, where $U_{w}:=U \cap\left(U^{-}\right)^{w}$ is the intersection of $U$ with the $\dot{w}$-conjugate of the opposite unipotent subgroup, $\left(U^{-}\right)^{w}:=\dot{w}^{-1} U^{-} \dot{w}$ (cf. [2, Thm. 14.12]). Note that $U_{w}$ is normalized by $T$ because both $U$ and $\left(U^{-}\right)^{w}$ are. We write $B g=B \dot{w} u$ for some $w \in W$ and $u \in U_{w}$, and we assume $B g$ is stabilized by the right action of $t \in T^{\prime}$. Observe

$$
\dot{w} u t=t^{\dot{w}^{-1}} \cdot \dot{w} \cdot u^{t}
$$

where $t^{\dot{w}^{-1}}:=\dot{w} t \dot{w}^{-1} \in T \subseteq B$ and $u^{t}:=t^{-1} u t \in U_{w}$. Since $t$ stabilizes $B g$, there exists some $b \in B$ such that $b \dot{w} u=t^{\dot{w}^{-1}} \cdot \dot{w} \cdot u^{t}$. Since $\phi$ is an isomorphism, it must be that $u=u^{t}$, $b=t^{\dot{w}^{-1}}$, and $\dot{w} u t=t^{\dot{w}^{-1}} \cdot \dot{w} u$. From this equality, it is clear that $t$ acts on the fibre of $\mathscr{L}(\chi)$ via multiplication by $\chi\left(t^{\dot{w}^{-1}}\right)=(\chi \cdot w)(t)$.

The following lemma will be the inductive step in the argument showing that for any $G$-variety $Z$ with a $G$-linearized ample line bundle, the variety $Y:=Z \times(B \backslash G)^{\text {rank } T}$ admits a $G$-linearized ample line bundle for which $Y_{T}^{s s}=Y_{T}^{s}$ and all points in $Y$ projecting to a stable point of $Z$ are stable in $Y$.

Lemma 2.1.2. Let $\mathscr{L}_{Z}$ be a $G$-linearized ample line bundle on a projective $G$-variety $Z$. If $r>0$ is the maximum rank of a subtorus of $T$ that stabilizes a strictly semi-stable point in $Z_{T}^{s s s}$, then there is a character $\chi \in \Lambda^{*}(T)$ and an integer $n \gg 0$ so that the induced right $G$-linearized line bundle $\mathscr{L}_{Z}^{\otimes n} \otimes \mathscr{L}(\chi)$ on $Z \times B \backslash G$ is ample and has the following properties:

(1) The subtori of $T$ that stabilize points in $(Z \times B \backslash G)_{T}^{\text {sss }}$ are at most rank $r-1$.

(2) A point $p \in Z \times B \backslash G$ is stable (resp. unstable) whenever $\pi(p)$ is so, where $\pi: Z \times B \backslash G \rightarrow$ $Z$ is projection onto the first factor and the notion of stability is taken with respect to either the $G$ - or the T-action.

Proof. Let $T_{1}, \ldots, T_{n}$ denote the positive-dimensional subtori of $T$ occurring as the connected components of stabilizers of points $z \in Z_{T}^{s s s}$. Let $H_{i}:=\Lambda^{*}\left(T / T_{i}\right) \subset \Lambda^{*}(T)$ denote the subgroup of $T$-characters vanishing on $T_{i}$. Since $T_{i}$ is positive dimensional, the inclusion $H_{i} \subsetneq \Lambda^{*}(T)$ is strict. Choose an integral weight $\chi$ in the interior of the positive Weyl chamber of $\Lambda^{*}(T)$ avoiding the $W$-orbit of any $H_{1}, \ldots, H_{n}$. By Lemma 2.1.1, $\mathscr{L}(\chi)$ is a $G$-linearized ample line bundle on $B \backslash G$.

By choosing $n$ large enough, one can derive from the Hilbert-Mumford criterion (Thm. 1.1.4), without too much effort, that the linearized line bundle $\mathscr{L}_{Z}^{\otimes n} \otimes \mathscr{L}(\chi)$ satisfies: 
- $p \in Z \times B \backslash G$ is stable if $\pi(p)$ is stable,

- $p \in Z \times B \backslash G$ is unstable if $\pi(p)$ is unstable,

for stability with respect to either the $G$ - or the $T$-action. This also follows immediately from a more general theorem of Reichstein (cf. [26, Thm. 2.1]). As a consequence, any strictly semi-stable point $p \in(Z \times B \backslash G)_{T}^{s s}$ must sit above a strictly semi-stable point $\pi(p) \in Z_{T}^{s s}$.

If $T^{\prime}$ is a torus stabilizing some point $(z, B g) \in(Z \times B \backslash G)_{T}^{s s s}$, then $z \in Z_{T}^{s s s}$. Therefore $T^{\prime} \subseteq T_{i}$ for some $1 \leq i \leq n$, and the weight of the action of $T^{\prime}$ on the fibre of $\mathscr{L}_{Z}$ over $z$ is 0 . Since $T^{\prime}$ stabilizes $B g$, Lemma2.1.1 implies the weight of the action of $T^{\prime}$ on the fibre of $\mathscr{L}(\chi)$ over $B g$ is $\chi \cdot w$ for some $w \in W$. Therefore, the weight of the action of $T^{\prime}$ on the fibre of $\mathscr{L}_{Z}^{\otimes n} \otimes \mathscr{L}(\chi)$ over $(z, B g)$ is $0+\left.(\chi \cdot w)\right|_{T^{\prime}}$. Since $(z, B g)$ is strictly semi-stable, this weight must be 0 and so $T^{\prime} \subseteq \operatorname{ker}\left(\left.(\chi \cdot w)\right|_{T_{i}}\right)$. Our choice of $\chi$ was such that $\left.(\chi \cdot w)\right|_{T_{i}} \neq 0 \in \Lambda^{*}\left(T_{i}\right)$ for any $w \in W$, and thus the rank of $T^{\prime}$ is at most the rank of $\operatorname{ker}\left(\left.(\chi \cdot w)\right|_{T_{i}}\right)$, which is $r-1$.

Proposition 2.1.3. If $Z$ is a projective $G$-variety with a $G$-linearized ample line bundle then for $r:=\operatorname{rank} T$, the variety $Y:=Z \times(B \backslash G)^{r}$ admits a G-linearized ample line bundle for which

(i) $Y^{s s}=Y^{s}$, and

(ii) $Z^{s} \times(B \backslash G)^{r} \subseteq Y^{s s} \subseteq Z^{s s} \times(B \backslash G)^{r}$,

for both $T$ - and $G$ - (semi-)stability.

Proof. We prove the results for $T$-stability, and then the result for $G$-stability will follow (cf. Thm.1.1.4).

Recursively applying Lemma 2.1.2, we obtain a $G$-linearized line bundle on $Z \times(B \backslash G)^{r}$ for which no $T$-strictly semi-stable points have positive dimensional $T$-stabilizers. Hence, all $T$-semi-stable points are $T$-stable, proving (i). Lemma 2.1.2(2) guarantees (ii).

2.2. Integration. Here we prove that the integration of Chow classes and their lifts on GIT quotients of a singular variety which equivariantly embeds into a nonsingular variety can be related to the integration of Chow classes and lifts on an auxiliary variety constructed as in the previous subsection. We begin with an easy lemma describing how lifting Chow classes commutes with proper pushes-forward.

Lemma 2.2.1. If $\pi: X \rightarrow Y$ is an equivariant proper morphism of $G$-varieties admitting $G$ linearized ample line bundles so that $X_{T}^{s s}=X_{T}^{s}, Y_{T}^{s s}=Y_{T}^{s}$, and $\pi^{-1}\left(Y_{T}^{s s}\right)=X_{T}^{s s}$, then for any Chow class $\sigma \in A_{*}(X / / G)_{\mathbb{Q}}$ and any lift $\tilde{\sigma} \in A_{*}(X / / T)_{\mathbb{Q}}$, the push-forward $\pi_{*} \tilde{\sigma} \in A_{*}(Y / / T)_{\mathbb{Q}}$ is a lift of the Chow class $\pi_{*} \sigma \in A_{*}(Y / / G)_{\mathbb{Q}}$.

Proof. The Hilbert-Mumford criterion (Thm. 1.1.4 implies that $\pi^{-1}\left(Y_{G}^{s s}\right)=X_{G}^{s s}$, yielding the following fibre square,

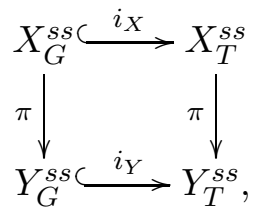

where the horizontal arrows are open immersions, and the vertical arrows are proper morphisms. By Definition 1.2.1, the Chow classes $\sigma, \tilde{\sigma}$ correspond to classes $\sigma \in A_{*}\left(\left[X_{G}^{s s} / G\right]\right)_{\mathbb{Q}}$ and $\tilde{\sigma} \in$ $A_{*}\left(\left[X_{T}^{s s} / T\right]\right)_{\mathbb{Q}}$ satisfying $i_{X}^{*} \tilde{\sigma}=f_{X}^{*} \sigma$, where $f_{X}:\left[X_{G}^{s s} / G\right] \rightarrow\left[X_{G}^{s s} / T\right]$ is induced by the inclusion $T \subseteq G$.

All that must be verified is whether

$$
i_{Y}^{*}\left(\pi_{*} \tilde{\sigma}\right) \stackrel{?}{=} f_{Y}^{*}\left(\pi_{*} \sigma\right)
$$


where the morphism $f_{Y}:\left[Y_{G}^{s s} / G\right] \rightarrow\left[Y_{G}^{s s} / T\right]$ is induced by the inclusion $T \subseteq G$. Since the above diagram is a fibre square, there is the commutativity $i_{Y}^{*} \circ \pi_{*}=\pi_{*} \circ i_{X}^{*}$ (cf. [11, Prop. 1.7]). Therefore,

$$
\begin{aligned}
i_{Y}^{*}\left(\pi_{*} \tilde{\sigma}\right) & =\pi_{*}\left(i_{X}^{*} \tilde{\sigma}\right) \\
& =\pi_{*}\left(f_{X}^{*} \sigma\right),
\end{aligned}
$$

since $\tilde{\sigma}$ is a lift of $\sigma$. It remains to show that $\pi_{*} \circ f_{X}^{*}=f_{Y}^{*} \circ \pi_{*}$, which follows from the fact that the commutative diagram of Deligne-Mumford stacks,

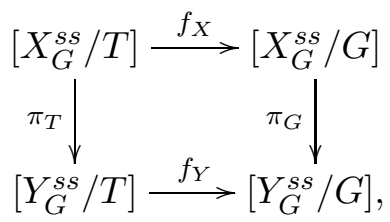

is a fibre square, as demonstrated by the following lemma.

Lemma 2.2.2. If $H \subseteq G$ is a closed subgroup of $G$ and $f: Y \rightarrow Z$ is an equivariant morphism of $G$-varieties, then $f$ induces the following 2-Cartesian diagram of quotient stacks:

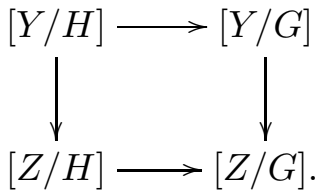

Proof. To begin, we recall that for a closed subgroup $H \subseteq G$ and any $G$-variety $U$, there is a Morita equivalence, corresponding to the isomorphism of quotients stacks

$$
[U / H] \cong\left[\left(U \times{ }_{H} G\right) / G\right],
$$

given by the operation $-\times_{H} G$ that converts an $H$-torsor mapping to $U$ into a $G$-torsors mapping to $U \times_{H} G$.

Note how the automorphism of $U \times G$ given by the rule $(u, g) \mapsto\left(u g, g^{-1}\right)$ intertwines the right action of $H$ on the $G$-factor with the right action given by the rule $(u, g) \cdot h:=\left(u h, h^{-1} g\right)$. This induces a $G$-equivariant isomorphism

$$
U \times G / H \cong U \times_{H} G,
$$

where the $G$-action on $U \times G / H$ is given by the rule $(u, g H) \cdot \hat{g}=\left(u \hat{g}, \hat{g}^{-1} g H\right)$, and the $G$-action on $U \times_{H} G$ by the rule $(u, g) \cdot \hat{g}=(u, g \hat{g})$. Combined with (2.2.4), this gives an isomorphism of quotient stacks

$$
[(U \times G / H) / G] \cong[U / H]
$$

Under this identification, the natural morphism $[U / H] \rightarrow[U / G]$ induced by the inclusion $H \subseteq G$ corresponds to the morphism $[(U \times G / H) / G] \rightarrow[U / G]$ induced by the $G$-equivariant projection $U \times G / H \rightarrow U$. Finally, since the $G$-equivariant diagram

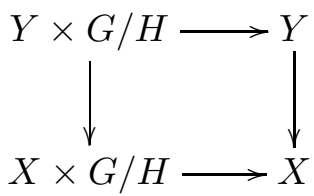

is Cartesian, so too will be the corresponding diagram of quotient stacks in the 2-category of stacks. 
We are now prepared to prove the main result of this subsection.

Proposition 2.2.6. Let $j: X \hookrightarrow Z$ be an equivariant embedding of $G$-varieties, and let $\mathscr{L}$ be a $G$ linearized ample line bundle on $Z$. There is a G-linearized ample line bundle on $Y:=Z \times(B \backslash G)^{r}$, where $r:=\operatorname{rank} T$, for which $Y_{T}^{s s}=Y_{T}^{s}$ and furthermore, if $X_{T}^{s s}=X_{T}^{s}$ for the induced $G$ linearized line bundle $j^{*} \mathscr{L}$ on $X$, then for any $\sigma \in A_{*}(X / / G)_{\mathbb{Q}}$ with lift $\tilde{\sigma} \in A_{*}(X / / T)_{\mathbb{Q}}$, there is a class $\tau \in A_{*}(Y / / G)_{\mathbb{Q}}$ with lift $\tilde{\tau} \in A_{*}(Y / / T)_{\mathbb{Q}}$ so that

(1) $\int_{X / / G} \sigma=\int_{Y / / G} \tau$, and

(2) $\int_{X / / T} c \frown(\Delta \frown \tilde{\sigma})=\int_{Y / / T} c \frown(\Delta \frown \tilde{\tau}), \forall c \in A^{*}(X / / T)_{\mathbb{Q}}$.

Proof. Let $\mathscr{M}$ denote the $G$-linearized ample line bundle on $Y$ prescribed in Proposition 2.1.3 Define the variety $\hat{X}:=X \times(B \backslash G)^{r}$ and the embedding $\hat{j}=j \times$ id $: \hat{X} \hookrightarrow Y$. Endow the line bundle $\hat{j}^{*} \mathscr{M}$ on $\hat{X}$ with the $G$-linearization induced from that on $\mathscr{M}$. Proposition 2.1.3 guarantees that $Y_{T}^{s s}=Y_{T}^{s}$, and hence $\hat{X}_{T}^{s s}=\hat{X}_{T}^{s}$. Moreover, it also shows that $\hat{j}^{-1}\left(Y_{T}^{s s}\right)=\hat{X}_{T}^{s s}$ and $\pi^{-1}\left(X_{T}^{s s}\right)=\hat{X}_{T}^{s s}$, for $\pi: \hat{X} \rightarrow X$ the projection morphism. Since $\left.\pi\right|_{X_{G}^{s s}}$ is surjective, there exists a class $\rho \in A_{*}(\hat{X} / / G)_{\mathbb{Q}}$ such that $\pi_{*} \rho=\sigma$. Let $\tilde{\rho} \in A_{*}(\hat{X} / / T)_{\mathbb{Q}}$ be a lift of the Chow class $\rho$ for which $\pi_{*}(\tilde{\rho})=\tilde{\sigma}$, which is possible because $\left.\pi\right|_{X_{T}^{s s}}$ is surjective. By Lemma2.2.1 $\tilde{\tau}:=\hat{j}_{*} \tilde{\rho}$ is a lift of the class $\tau:=\hat{j}_{*} \rho$.

By the functoriality of the Chow groups under proper push-forward, the commutative diagram,

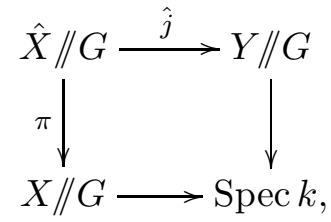

shows that $\int_{X / / G} \sigma=\int_{Y / / G} \tau$, proving (1). The equality in (2) follows in the same way from an analogous diagram involving $T$-quotients, recalling that the projection-formula guarantees the compatibility of the operational Chow class $c \frown \Delta$ under pushes-forward.

\subsection{Application.}

Proposition 2.3.1. Let $X$ be a projective variety over a field $k$ with a $G$-linearized ample line bundle for which $X_{T}^{s s}=X_{T}^{s}$. If $\tilde{\sigma}_{0}, \tilde{\sigma}_{1} \in A_{*}(X / / T)_{\mathbb{Q}}$ are two lifts of the same class $\sigma \in A_{*}(X / / G)_{\mathbb{Q}}$, then for any operational Chow class $c \in A^{*}(X / / T)_{\mathbb{Q}}$,

$$
\int_{X / / T} c \frown\left(\Delta \frown \tilde{\sigma}_{0}\right)=\int_{X / / T} c \frown\left(\Delta \frown \tilde{\sigma}_{1}\right) .
$$

Proof. Embed the singular $X$ into the smooth variety $\mathbb{P}(V)$ via some high tensor power of the given $G$-linearized line bundle. Construct the smooth $G$-variety $Y$ as in Proposition 2.2.6 by taking $Z:=\mathbb{P}(V)$. The proposition guarantees that any two lifts $\tilde{\sigma}_{0}$ and $\tilde{\sigma}_{1}$ of a class $\sigma \in A_{*}(X / / G)_{\mathbb{Q}}$ have analogues $\tilde{\tau}_{0}$ and $\tilde{\tau}_{1}$ both lifting a class $\tau \in A_{*}(Y / / G)_{\mathbb{Q}}$ and satisfying

$$
\int_{X / / T} c \frown\left(\Delta \frown \tilde{\sigma}_{i}\right)=\int_{Y / / T} c \frown\left(\Delta \frown \tilde{\tau}_{i}\right),
$$

for $i=0,1$. The result immediately follows from the smooth case (cf. Cor. 1.5.4). 


\section{INVARIANCE OF THE GIT INTEGRATION RATIO}

The goal of this section is to prove Theorem 0.3 that for a projective $G$-variety $X$ over a field $k$ and a $G$-linearized ample line bundle for which $X_{T}^{s s}=X_{T}^{s}$, the following ratio is an invariant of the group $G$ :

Definition 3.0.1. Assume $X$ is a projective $G$-variety with a $G$-linearized ample line bundle $\mathscr{L}$ for which $X_{T}^{s s}=X_{T}^{s}$, and $\sigma \in A_{0}(X / / G)$ is a 0 -cycle satisfying $\int_{X / / G} \sigma \neq 0$. We define the $G I T$ integration ratio to be

$$
r_{G, T}^{X, \sigma}:=\frac{\int_{X / / T} c_{\mathrm{top}}(\mathfrak{g} / \mathfrak{t}) \frown \tilde{\sigma}}{\int_{X / / G} \sigma},
$$

where $\tilde{\sigma}$ is some lift of the class $\sigma$.

Note that the GIT integration ratio is well-defined, as can be seen by taking $c=(-1)^{|\Phi| / 2} \Delta$ and applying Proposition 2.3.1, We shall next prove in stages that $r_{G, T}^{X, \sigma}$ is independent of the choice of Chow class $\sigma \in A_{0}(X / / G)_{\mathbb{Q}}$, the choice of maximal torus $T$, and even the choices of $G$-linearized ample line bundle $\mathscr{L}$ and projective $G$-variety $X$.

\subsection{Chow class.}

Lemma 3.1.1. The GIT integration ratio $r_{G, T}^{X}:=r_{G, T}^{X, \sigma}$ is independent of the choice of Chow class $\sigma \in A_{0}(X / / G)_{\mathbb{Q}}$.

Proof. The definition of $r_{G, T}^{X, \sigma}$ is independent of the algebraic equivalence class of $\sigma$, since numerical equivalence is coarser than algebraic equivalence. Let $B_{*}(-)$ denote the quotient of the Chow group $A_{*}(-)$ by the relation of algebraic equivalence (cf. [11, $\left.\S 10.3\right]$ ). All connected projective schemes are algebraically connected, i.e. there is a connected chain of (possibly singular) curves connecting any two closed points. Therefore, $B_{0}(X / / G)_{\mathbb{Q}}=\mathbb{Q}$, and the result follows since $r_{G, T}^{X, \sigma}$ is invariant under the multiplication of $\sigma$ by a nonzero scalar.

3.2. Maximal torus. We begin by reducing to the case where $k=\bar{k}$ is an algebraically closed field.

Lemma 3.2.1. Let $X$ be a projective $G$-variety over a field $k$ equipped with a $G$-linearized ample line bundle $\mathscr{L}$ for which $X_{T}^{s s}=X_{T}^{s}$. For any field extension $K / k$, the GIT integration ratios are equal,

$$
r_{G, T}^{X}=r_{G_{K}, T_{K}}^{X},
$$

where $X_{K}, G_{K}$, and $T_{K}$ denote the base changes from $k$ to $K$ of $X, G$, and $T$, respectively.

Proof. By [12, Prop. 1.14], $X_{G}^{s s} \times K=\left(X_{K}\right)_{G_{K}}^{s s}$ for the induced $G_{K}$-linearization on $\mathscr{L}_{K}$ over $X_{K}$ (and similarly with $T$ replacing $G$ ). Since field extensions are flat, taking the uniform categorical quotient commutes with extending the field, so $(X / / G)_{K} \cong X_{K} / / G_{K}$ and $(X / / T)_{K} \cong X_{K} / / T_{K}$. The result can then be deduced from the fact that the degree of a Chow class is invariant under field extension (cf. [11, Ex. 6.2.9]).

Lemma 3.2.2. The GIT integration ratio $r_{G}^{X}:=r_{G, T}^{X}$ does not depend on the choice of maximal torus $T$.

Proof. By Lemma 3.2.1, we may assume that $k=\bar{k}$. For any two maximal tori $T, T^{\prime} \subseteq G$, there exists some $g \in G(\bar{k})$ such that $T^{\prime}=g T g^{-1}$. By assumption, $G$ acts linearly on the projective variety $X$. Consider the map $\psi: T \rightarrow T^{\prime}$ given by $t \mapsto g t g^{-1}$, and the map $\Psi: X \rightarrow X$ given by 
$x \mapsto x \cdot g^{-1}$. The pair of maps $(\psi, \Psi)$ show that the actions $\sigma: X \times T \rightarrow X$ and $\sigma^{\prime}: X \times T^{\prime} \rightarrow X$ are isomorphic: $\Psi(x) \cdot \psi(t)=\Psi(x \cdot t)$. By the Hilbert-Mumford numerical criterion (Thm. 1.1.4), $X_{T^{\prime}}^{s s}=\Psi\left(X_{T}^{s s}\right)$, and hence the following square is commutative,

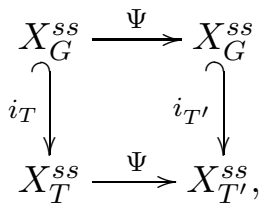

where the vertical arrows are open immersions and the horizontal arrows are isomorphisms. From this diagram, it is clear that for any lift $\tilde{\sigma} \in A_{*}(X / / T)_{\mathbb{Q}}$ of a Chow class $\sigma \in A_{0}(X / / G)_{\mathbb{Q}}$, the push-forward $\Psi_{*} \tilde{\sigma} \in A_{*}\left(X / / T^{\prime}\right)_{\mathbb{Q}}$ is a lift of $\Psi_{*} \sigma=\sigma$. We use the pairs $(\sigma, \tilde{\sigma})$ and $\left(\sigma, \Psi_{*} \tilde{\sigma}\right)$ to compute the GIT integration ratios. Since $\Psi$ is an isomorphism, the classes $c_{\text {top }}(\mathfrak{g} / \mathfrak{t}) \frown \tilde{\sigma}$ and $\Psi_{*}\left(c_{\text {top }}(\mathfrak{g} / \mathfrak{t}) \frown \tilde{\sigma}\right)=c_{\text {top }}\left(\mathfrak{g} / \mathfrak{t}^{\prime}\right) \frown \Psi_{*} \tilde{\sigma}$ have the same degree, where $\mathfrak{t}^{\prime}$ denotes the Lie algebra of $T^{\prime}$. Therefore, the ratios $r_{G, T}^{X}$ and $r_{G, T^{\prime}}^{X}$ are equal.

\subsection{Linearized line bundle and variety.}

Lemma 3.3.1. The GIT integration ratio $r_{G}:=r_{G}^{X}$ does not depend on either the choice of $G$ linearized ample line bundle $\mathscr{L}$ or the projective $G$-variety $X$.

Proof. For each $i=1,2$, let $X_{i}$ be a projective $G$-variety with a $G$-linearized ample line bundle $\mathscr{L}_{i}$ for which $\left(X_{i}\right)_{T}^{s s}=\left(X_{i}\right)_{T}^{s}$. Some high tensor power of $\mathscr{L}_{i}$ defines a $G$-equivariant embedding $j_{i}$ : $X_{i} \hookrightarrow \mathbb{P}\left(V_{i}\right)$ for some $G$-representation $V_{i}$. Consequently, one derives an equivariant embedding $\hat{j}_{i}: X_{i} \hookrightarrow \mathbb{P}\left(V_{1} \oplus V_{2}\right)$ from the embedding $j_{i}$, simply by setting the extraneous coordinates to 0 . Moreover, the embeddings $\hat{j}_{i}$ are compatible with the $G$-linearization on the line bundle $\mathcal{O}(1)$ on $\mathbb{P}\left(V_{1} \oplus V_{2}\right)$, induced from the direct sum representation of $G$ on $V_{1} \oplus V_{2}$, and the given $G$ linearizations on the line bundles $\mathscr{L}_{i}$. By Proposition 2.2.6 for any classes $\sigma_{i} \in A_{0}\left(X_{i} / / G\right)_{\mathbb{Q}}$, there are classes $\tau_{i} \in A_{0}(Y / / G)_{\mathbb{Q}}$, for some smooth, projective $G$-variety $Y$ with a $G$-linearized ample line bundle for which $Y_{T}^{s s}=Y_{T}^{s} \neq \emptyset$ and $r_{G}^{Y, \tau_{i}}=r_{G}^{X_{i}, \sigma_{i}}$. By Lemma 3.1.1 $r_{G}^{Y, \tau_{1}}=r_{G}^{Y, \tau_{2}}$ and therefore $r_{G}^{X_{1}, \sigma_{1}}=r_{G}^{X_{2}, \sigma_{2}}$.

The above lemmas combine to demonstrate Theorem 0.3

\section{DiReCT PRODUCTS AND CENTRAL ISOGENIES}

In this section, we prove that the GIT integration ratio behaves well with respect to the group operations of direct product and central isogeny. The results of this section combine to prove Theorem 0.4 .

Proposition 4.1. If $G_{1}, G_{2}$ are two reductive groups over a field $k$, then $r_{G_{1} \times G_{2}}=r_{G_{1}} \cdot r_{G_{2}}$.

Proof. For each $i=1,2$, choose a projective $G_{i}$-variety $X_{i}$ and a $G_{i}$-linearized ample line bundle $\mathscr{L}_{i}$. Let $T_{i} \subseteq G_{i}$ denote a maximal torus. Clearly $G_{1} \times G_{2}$ acts on $X_{1} \times X_{2}$, and $\mathscr{L}_{1} \otimes \mathscr{L}_{2}$ is a $G_{1} \times G_{2}$-linearized ample line bundle for which $\left(X_{1} \times X_{2}\right)_{G_{1} \times G_{2}}^{s s}=\left(X_{1}\right)_{G_{1}}^{s s} \times\left(X_{2}\right)_{G_{2}}^{s s}$. Let $\sigma_{i} \in A_{0}^{G_{i}}\left(\left(X_{i}\right)_{G_{i}}^{s s}\right)$, and consider $\sigma:=\sigma_{1} \times \sigma_{2} \in A_{0}^{G_{1} \times G_{2}}\left(\left(X_{1}\right)_{G_{1}}^{s s} \times\left(X_{2}\right)_{G_{2}}^{s s}\right)$. Also, take $\tilde{\sigma}:=\tilde{\sigma}_{1} \times \tilde{\sigma}_{2}$, where each $\tilde{\sigma}_{i}$ lifts $\sigma_{i}$. By Theorem 0.3 , it suffices to calculate the GIT integration ratio for $G_{1} \times G_{2}$ using the classes $\sigma$ and $\tilde{\sigma}$. The degree of a product of two classes is the product of the degrees, and so the result follows since the isomorphism $[\operatorname{Spec} k / T] \cong\left[\operatorname{Spec} k / T_{1}\right] \times_{k}\left[\operatorname{Spec} k / T_{2}\right]$ identifies $c_{\text {top }}\left(\mathfrak{g}_{1} / \mathfrak{t}_{1}\right) \times c_{\text {top }}\left(\mathfrak{g}_{2} / \mathfrak{t}_{2}\right)$ with $c_{\text {top }}(\mathfrak{g} / \mathfrak{t})$. 
Now we prove that $r_{G}$ is invariant under central isogeny. This will completing the proof of Theorem 0.4 (since the GIT integration ratio of a torus is clearly 1 ).

Proposition 4.2. If $G \rightarrow \bar{G}$ is a central isogeny of connected reductive groups, then $r_{G}=r_{\bar{G}}$.

Proof. Since the kernel of $G \rightarrow G$ is a finite group scheme of order $d<\infty$, we may use the same $\bar{G}$-variety $X$ on which to calculate both ratios $r_{G}$ and $r_{\bar{G}}$. These ratio will therefore agree, since the numerator and the denominator of the ratio $r_{G}$ will only differ from those of $r_{\bar{G}}$ by a factor of $d$.

\section{Groups of TyPe $\mathbf{A}_{n}$}

We compute the GIT integration ratio $r_{G}$ for $G=P G L(n)$, and use this to prove Corollary 0.5

Proposition 5.1. The GIT integration ratio for the group $G=P G L(n)$ defined over any field is

$$
r_{G}=|W|=n !
$$

Proof. Let $G L(n)$ act on $\mathbf{M}_{n}$, the vector space of $n \times n$ matrices with $k$-valued entries, via right multiplication of matrices. This induces a dual right-action of $G L(n)$ on $\mathbf{M}_{n}^{*}$ and hence rightactions of $G L(n), S L(n)$, and $P G L(n)$ on $\mathbb{P}\left(\mathbf{M}_{n}\right)$, the projective space of lines in $\mathbf{M}_{n}^{*}$. Choose the $P G L(n)$-linearization on $\mathcal{O}_{\mathbb{P}\left(\mathbf{M}_{n}\right)}(n)$ induced from these representations on $\mathbf{M}_{n}$. Let $T \subseteq$ $G L(n), S \subseteq S L(n)$, and $\bar{T} \subseteq P G L(n)$ denote the diagonal maximal tori. Because of the isogeny $S L(n) \rightarrow P G L(n)$, the $P G L(n)$-stability loci (resp. $\bar{T}$-stability loci) are equal to the analogous $S L(n)$-stability loci (resp. $S$-stability loci), which we proceed to describe.

A basis of $\mathbf{M}_{n}$ is given by the matrices $e_{i j}$, each defined by its unique nonzero entry of 1 in the $(i, j)$ th position. Moreover, $e_{i j}$ is a weight vector of weight $\chi_{j} \in \Lambda^{*}(T)$, with $\chi_{j}$ defined by the rule

$$
\left(\begin{array}{cccc}
t_{1} & & & \\
& t_{2} & & \\
& & \ddots & \\
& & & t_{n}
\end{array}\right) \mapsto t_{j} .
$$

Notice that $\chi_{1}, \ldots, \chi_{n}$ is a basis of the character group $\Lambda^{*}(T)$, and $\Lambda^{*}(S)$ is spanned by the restriction of these characters $\left.\chi_{i}\right|_{S}$, which are only subject to the relation $\left.\chi_{n}\right|_{S}=-\left.\sum_{i=1}^{n-1} \chi_{i}\right|_{S}$. Therefore, the characters $\left.\chi_{1}\right|_{S}, \ldots,\left.\chi_{n}\right|_{S}$ form the vertices of a simplex centered at the origin in the vector space $\Lambda^{*}(S) \otimes \mathbb{Q} \cong \mathbb{Q}^{n-1}$. From the Hilbert-Mumford criterion (Thm. 1.1.4), one quickly concludes that the unstable locus $\mathbb{P}\left(\mathbf{M}_{n}\right)_{\bar{T}}^{u n}$ is the set of all points $x \in \mathbb{P}\left(\mathbf{M}_{n}\right)$ such that the matrix $e_{i j}(x)$ has a column with all entries 0 ; all other points are $\bar{T}$-stable. Such stable points have trivial $\bar{T}$-stabilizers, and so the $\bar{T}$-quotient is easily seen to be

$$
\mathbb{P}\left(\mathbf{M}_{n}\right) / / \bar{T} \cong\left[\mathbb{P}\left(\mathbf{M}_{n}\right)_{\bar{T}}^{s s} / \bar{T}\right] \cong\left(\mathbb{P}^{n-1}\right)^{n} .
$$

Furthermore, if we denote by $\mathbf{M}_{n}^{\circ} \cong\left(\mathbb{A}^{n} \backslash 0\right)^{n}$ the preimage in $\mathbf{M}_{n} \backslash 0$ of $\mathbb{P}\left(\mathbf{M}_{n}\right)_{\bar{T}}^{s s}$, there is also an identification

$$
\mathbb{P}\left(\mathbf{M}_{n}\right) / / \bar{T} \cong\left[\mathbf{M}_{n}^{\circ} / T\right]
$$

From this later description, we see immediately that the rational Chow ring is given as an $A^{*}(B T)_{\mathbb{Q}} \cong$ $\operatorname{Sym}_{\mathbb{Q}}^{*} \Lambda^{*}(T)$-algebra by

$$
A^{*}\left(\mathbb{P}\left(\mathbf{M}_{n}\right) / / \bar{T}\right)_{\mathbb{Q}} \cong \mathbb{Q}\left[\chi_{1}, \ldots, \chi_{n}\right] /\left(\chi_{1}^{n}, \ldots, \chi_{n}^{n}\right) .
$$

In this ring, the class of a point is clearly $\prod_{i=1}^{n} \chi_{i}^{n-1}$, and therefore the degree of a Chow cycle is given by the coefficient of this monomial. 
The $P G L(n)$-stable locus then comprises the set of $x \in \mathbb{P}\left(\mathbf{M}_{n}\right)$ such that the matrix $e_{i j}(x)$ is of full-rank; this comprises the dense $P G L(n)$ orbit given by the inclusion $P G L(n) \subseteq \mathbb{P}\left(\mathbf{M}_{n}\right)$. The stabilizer of this orbit is trivial, and hence

$$
\mathbb{P}\left(\mathbf{M}_{n}\right) / / P G L(n) \cong\left[\mathbb{P}\left(\mathbf{M}_{n}\right)_{P G L(n)}^{s s} / P G L(n)\right] \cong \operatorname{Spec} k .
$$

The Chow ring of this variety is simply $\mathbb{Q}$ in degree 0 , and by choosing $\sigma=1$, the fundamental class, the computation of the GIT integration ratio $r_{G}$ is reduced to evaluating $r_{G}=\int_{\mathbb{P}\left(\mathbf{M}_{n}\right) / / T} c_{\text {top }}(\mathfrak{g} / \mathfrak{t})$.

The class $c_{\text {top }}(\mathfrak{g} / \mathfrak{t}) \in A^{*}(B \bar{T})$ is the product of all the roots of $P G L(n)$; these are of the form $\alpha_{i j}:=\chi_{i}-\chi_{j} \in \operatorname{Sym}^{*} \Lambda^{*}(T) \cong A^{*}(B \bar{T})$, for $1 \leq i \neq j \leq n$. Therefore $r_{G}$ equals the coefficient of the monomial $\prod_{i=1}^{n} \chi_{i}^{n-1}$ in the expansion of $\prod_{i \neq j}\left(\chi_{i}-\chi_{j}\right)$. We can compute this coefficient as follows. The class $c_{\text {top }}(\mathfrak{g} / \mathfrak{t})$ may be alternatively expressed as

$$
\prod_{i \neq j}\left(\chi_{i}-\chi_{j}\right)=(-1)^{n(n-1) / 2}\left(\operatorname{det} M_{V}\right)^{2}
$$

where $\operatorname{det} M_{V}$ is the determinant of the Vandermonde matrix

$$
M_{V}:=\left(\begin{array}{ccccc}
1 & \chi_{1} & \chi_{1}^{2} & \cdots & \chi_{1}^{n-1} \\
1 & \chi_{2} & \chi_{2}^{2} & \cdots & \chi_{2}^{n-1} \\
\vdots & \vdots & \vdots & \ddots & \vdots \\
1 & \chi_{n} & \chi_{n}^{2} & \cdots & \chi_{n}^{n-1}
\end{array}\right)
$$

By definition, $\operatorname{det} M_{V}=\sum_{\sigma \in S_{n}} \operatorname{sgn}(\sigma) \prod_{i=1}^{n} \chi_{i}^{\sigma(i)-1}$. In the Chow ring $A^{*}\left(\mathbb{P}\left(\mathbf{M}_{n}\right) / / \bar{T}\right)_{\mathbb{Q}}$, the products of monomials of the form $m_{\sigma}:=\prod_{i=1}^{n} \chi_{i}^{\sigma(i)-1}$ for $\sigma \in S_{n}$ are defined by the rule:

$$
m_{\sigma} \cdot m_{\sigma^{\prime}}= \begin{cases}\prod_{i=1}^{n} \chi_{i}^{n-1} & : \sigma(j)+\sigma^{\prime}(j)=n+1 ; \forall 1 \leq j \leq n \\ 0 & : \text { otherwise. }\end{cases}
$$

If $w_{0}:=(1 n)(2 n-1) \cdots(\lceil n / 2\rceil\lceil(n+1) / 2\rceil) \in S_{n}$ denote the longest element of the Weyl group $W=S_{n}$, then for each $\sigma \in S_{n}$, the permutation $\sigma^{\prime}$ defined as the composition $\sigma^{\prime}:=w_{0} \circ \sigma$ is the unique element of $S_{n}$ for which $m_{\sigma} \cdot m_{\sigma^{\prime}} \neq 0$. For such pairs $\left(\sigma, \sigma^{\prime}\right)$, the product of the signs satisfies $\operatorname{sgn}(\sigma) \cdot \operatorname{sgn}\left(\sigma^{\prime}\right)=\operatorname{sgn}\left(w_{0}\right)=(-1)^{\left(n^{2}-n\right) / 2}$. Therefore,

$$
\begin{aligned}
c_{\text {top }}(\mathfrak{g} / \mathfrak{t}) & =(-1)^{\left(n^{2}-n\right) / 2} \cdot \sum_{\sigma \in S_{n}}(-1)^{\left(n^{2}-n\right) / 2} \prod_{i=1}^{n} \chi_{i}^{n-1} \\
& =n ! \cdot \prod_{i=1}^{n} \chi_{i}^{n-1} .
\end{aligned}
$$

Thus, $r_{G}=n !=|W|$.

Having proved the above proposition, the proof of Corollary 0.5 is now anticlimactic:

Proof of Cor. 0.5 Combine Theorems 0.3 and 0.4 and Proposition 5.1

\section{GROUPS OF OTHER TYPES}

We conclude with a discussion of how to generalize Corollary 0.5 to arbitrary connected reductive groups, that is, how to prove Amplification 0.6 The argument will rely on Martin's original result [24. Thm. B'], and the discovery of an independent proof is left as an open question. 
Amplification 0.6. Let $G$ be a connected reductive group over a field $k$ and $T \subseteq G$ a maximal torus. For any $G$-linearized ample line bundle on a projective $G$-variety $X$ over $k$ satisfying $X_{T}^{s}=X_{T}^{s s}$ and any Chow class $\sigma \in A_{0}(X / / G)_{\mathbb{Q}}$ with lift $\tilde{\sigma} \in A_{*}(X / / T)_{\mathbb{Q}}$,

$$
\int_{X / / G} \sigma=\frac{1}{|W|} \int_{X / / T} c_{\mathrm{top}}(\mathfrak{g} / \mathfrak{t}) \frown \tilde{\sigma}
$$

Proof. We begin by pointing the reader to [27] as a reference on geometric invariant theory relative to a base. The base we will use is the spectrum of the ring $\mathbb{Z}_{(p)}$, the localization of $\mathbb{Z}$ at the prime $p$ equal to the characteristic of the base field $k$.

By Theorems 0.3 and 0.4 it suffices to verify $r_{G}^{X}=|W|$ on a single projective $G$-variety $X$ for each simple Chevalley group $G$. Each Chevalley group $G$ admits a model $G_{\mathbb{Z}}$ over the integers, with a split maximal torus $T_{\mathbb{Z}} \subseteq G_{\mathbb{Z}}$. We assert that there is a smooth projective $\mathbb{Z}_{(p)}$-scheme $X_{(p)}$ on which $G_{(p)}:=G_{\mathbb{Z}} \times_{\mathbb{Z}} \mathbb{Z}_{(p)}$ acts as well as a $G_{(p)^{-}}$-linearized ample line bundle for which all $G_{(p)^{-}}$ (resp. $T_{(p)^{-}}$) semi-stable points are stable and comprise an open locus that nontrivially intersects the closed fibre over $\mathbb{F}_{p}$. We justify this assertion briefly: Proposition 2.1.3 reduces the problem to finding some $\mathbb{Z}_{(p)}$-scheme for which there exist $G_{(p)}$-stable points in the closed fibre over $\mathbb{F}_{p}$; with the aid of the Hilbert-Mumford criterion, one discovers that many such schemes exist (e.g. take $\mathbb{P}\left(V_{\mathbb{Z}_{(p)}}^{\oplus n}\right)$ with $V_{\mathbb{Z}_{(p)}}^{\oplus n}$ a large multiple of a general irreducible $G_{(p)}$-representation).

Having chosen such an $X_{(p)}$, the technique of specialization (cf. [11, §20.3]) implies that the integral of relative 0 -cycles on $X_{(p)} / / G_{(p)}$ and $X_{(p)} / / T_{(p)}$ restricted to the generic fibre over $\mathbb{Q}$ is equal to the integral restricted to any closed fibre over $\mathbb{F}_{p}$. The ratio $r_{G}$ is independent under field extension by Lemma 3.2.1, and so this reduces the calculation of $r_{G}$ over the field $k$ to the computation of $r_{G_{\mathbb{C}}}$, where $G_{\mathbb{C}}:=G_{\mathbb{Z}} \times_{\mathbb{Z}} \mathbb{C}$. The Kirwan-Kempf-Ness theorem (cf. [22, §8] or [12, §8.2]) shows that in the analytic topology, the GIT quotient $X_{\mathbb{C}} / / G_{\mathbb{C}}$ is homeomorphic to the symplectic reduction of $X_{\mathbb{C}}$ by a maximal compact subgroup, and so Martin's theorem [24, Thm. $\left.\mathrm{B}^{\prime}\right]$ implies $r_{G_{\mathbb{C}}}=|W|$.

Question 6.1. What is a purely algebraic proof that $r_{G}=|W|$ for a general connected reductive group $G$ ?

In light of Theorems 0.3 and 0.4 , to answer Question 6.1 it suffices to verify $r_{G}=|W|$ for all simple groups $G$. Such a verification was done in $\$ 5$ for simple groups of type $\mathbf{A}_{n}$. Can $r_{G}$ be calculated (algebraically) for any other simple groups $G$ ?

\section{APPENDIX: CHOW GROUPS AND QUOTIENT STACKS}

Here we recall the basic properties of Chow groups for schemes and quotient stacks.

A.1. Chow groups. For a scheme $X$ defined over a field $k$, let $A_{i}(X)$ denote the $\mathbb{Z}$-module generated by $i$-dimensional subvarieties over $k$ modulo rational equivalence (cf. [11]). We call $A_{*}(X):=$ $\oplus_{i} A_{i}(X)$ the Chow group of $X$. To indicate rational coefficients, we write $A_{*}(X)_{\mathbb{Q}}:=A_{*}(X) \otimes \mathbb{Q}$.

For a scheme $X$ over a field $k$ and an algebraic group $G$ acting on $X$, the Chow group of the quotient stack $[X / G]$ is defined by Edidin and Graham in [7] to be the limit of Chow groups using Totaro's finite approximation construction (cf. [29]):

$$
A_{i}([X / G]):=A_{i-g+\ell}\left(X \times_{G} U\right),
$$

where $U$ is an open subset of an $\ell$-dimensional $G$-representation $V$ on which the $g$-dimensional group $G$ acts freely and whose complement $V \backslash U$ has sufficiently large codimension. It is a result of Edidin and Graham that this group is well-defined, independent of the presentation of the stack 
$[X / G]$ as a quotient, and recovers Gillet's original definition [14] of Chow groups on DeligneMumford stacks upon tensoring with $\mathbb{Q}$ (see $[7, \S 5]$ ). We may also think of $A_{i}[X / G]$ as the $G$ equivariant Chow group of $X$ to highlight the functoriality with respect to group homomorphisms, and we make use of the notation $A_{*}^{G}(X):=A_{*}([X / G])$.

One benefit of the stacky interpretation arises when $X$ admits a geometric invariant theory quotient $X / / G$. In this case, there is a coarse moduli morphism from the quotient stack $\left[X_{G}^{s s} / G\right]$ to $X / / G$ that induces an isomorphism of Chow groups with rational coefficients:

Theorem A.1.1 (Edidin-Graham, Gillet, Vistoli). If $X$ is a projective $G$-variety with a $G$-linearized ample line bundle for which $X_{G}^{s s}=X_{G}^{s}$, then the induced morphism $\phi:\left[X_{G}^{s s} / G\right] \rightarrow X / / G$ yields an isomorphism of rational Chow groups,

$$
\phi^{*}: A_{*}(X / / G)_{\mathbb{Q}} \rightarrow A_{*}([X / G])_{\mathbb{Q}} .
$$

Proof. See [7, Thm. 4] for a proof of the theorem, and the remark that follows it for a discussion of the prior results of Gillet [14] and Vistoli [31].

The Chow groups of quotient stacks are functorial with respect to the usual operations (e.g. flat pull-back, proper push-forward), and when $X$ is a smooth $n$-dimensional variety, there is an intersection product that endows these groups with the structure of a commutative ring with identity, graded by codimension and denoted by $A^{*}([X / G]):=A_{n-g-*}([X / G])$. Hence the Chow group of the stack $A_{*}([X / G])$ is naturally a module over the ring $A^{*}(B G)$ where $B G=[\operatorname{Spec} k / G]$. In the case $T=\mathbb{G}_{m}^{r}$ is a split torus of rank $r$,

$$
A^{*}(B T) \cong \operatorname{Sym} \Lambda^{*}(T) \cong \mathbb{Z}\left[\chi_{1}, \ldots, \chi_{r}\right],
$$

where $\chi_{1}, \ldots, \chi_{r}$ is some $\mathbb{Z}$-basis of $\Lambda^{*}(T)$. A character $\chi \in \Lambda^{*}(T)$ is equivalent to a line bundle $\mathscr{L}_{\chi}$ over $B T$ whose Chern class $c_{1}\left(\mathscr{L}_{\chi}\right) \in A^{*}(B T)$ corresponds to $\chi$ under the above isomorphism.

Not surprisingly, the structure of the Chow group $A_{*}([X / T])$ of the stacky quotient of $X$ by a torus $T$ is especially well-understood (see [4]).

Proposition A.1.2 (Brion). Let $X$ be a scheme with the action of a torus $T$ over an algebraically closed field $\bar{k}$. The T-equivariant Chow group $A_{*}^{T}(X)$ is generated as an $A^{*}(B T)$-module by the classes $[Y]$ associated to T-invariant closed subschemes $Y \hookrightarrow X$.

Proof. See [4, Thm. 2.1].

Moreover, there is a localization theorem useful for making calculations in $T$-equivariant Chow groups. The following version of the localization theorem will suffice for our purposes:

Theorem A.1.3 (Localization). Let $X$ be a smooth projective scheme with the action of a torus $T$ over an algebraically closed field $\bar{k}$, and let $i: X^{T} \rightarrow X$ denote the inclusion of the scheme of T-fixed points. Then the morphism

$$
i^{*}: A_{*}^{T}(X)_{\mathbb{Q}} \rightarrow A_{*}^{T}\left(X^{T}\right)_{\mathbb{Q}}
$$

is an injective $A^{*}(B T)$-algebra morphism. Furthermore, if $X^{T}$ consists of finitely many points, then the morphism

$$
i^{*}: A_{*}^{T}(X) \rightarrow A_{*}^{T}\left(X^{T}\right)
$$

of Chow groups with integer coefficients is injective as well.

Proof. See [4, Cor. 3.2.1]. 
A.2. Operational Chow groups. The $i$ th operational Chow group $A^{i}(X)$ is defined to be the group of "operations" $c$ that comprise a system of group homomorphisms $c_{f}: A_{*}(Y) \rightarrow A_{*-i}(Y)$ associated to morphisms of schemes $f: Y \rightarrow X$ and compatible with proper push-forward, flat pull-back, and the refined Gysin map (cf. [11, §17]). Similarly, Edidin and Graham define equivariant operational Chow groups $A_{G}^{i}(X)$ via systems of group homomorphisms $c_{f}^{G}: A_{*}^{G}(Y) \rightarrow A_{*-i}^{G}(Y)$ compatible with the $G$-equivariant analogues of the above maps (cf. [7, §2.6]).

The clearest examples of equivariant operational Chow classes are equivariant Chern classes $c_{i}(\mathscr{E})$ of $G$-linearized vector bundles $\mathscr{E}$ (i.e. Chern classes of vector bundles on $[X / G]$ ). Moreover, $A_{G}^{*}(X)$ equipped with composition forms an associative, graded ring with identity. When $X$ is smooth, there is a Poincare duality between the equivariant operational Chow group and the usual equivariant Chow group. For any operational Chow class $c=\left\{c_{f}^{G}\right\} \in A_{G}^{i}(X)$ and Chow class $\sigma \in A_{*}^{G}(Y)$, we introduce the following "cap product" notation:

$$
c \frown \sigma:=c_{f}^{G}(\sigma) \in A_{*-i}^{G}(Y) .
$$

Theorem A.2.4 (Poincaré duality). If $X$ is a smooth n-dimensional variety, then the map $A_{G}^{i}(X) \rightarrow$ $A_{n-i}^{G}(X)$ defined by $c \mapsto c \frown[X]$ is an isomorphism.

Proof. See [7, Prop. 4].

Remark A.2.5. When $X$ is a smooth n-dimensional variety, this allows us to write $A_{G}^{k}(X)$ to denote the codimension $k$ Chow group $A_{n-k}^{G}(X)$, without any ambiguity in notation. Furthermore, this identification forms an isomorphism of rings $A_{G}^{*}(X) \cong A_{n-*}^{G}(X)$, with the multiplication structure on $A_{n-*}^{G}(X)$ given by the intersection product.

\section{REFERENCES}

[1] S. Bloch, Lectures on algebraic cycles, 2nd ed., New Mathematical Monographs, vol. 16, Cambridge University Press, Cambridge, 2010.

[2] A. Borel, Linear algebraic groups, 2nd ed., Graduate Texts in Mathematics, vol. 126, Springer-Verlag, New York, 1991.

[3] M. Brion, Cohomologie équivariante des points semi-stables, J. Reine Angew. Math. 421 (1991), 125-140 (French).

[4] _ Equivariant Chow groups for torus actions, Transform. Groups 2 (1997), no. 3, 225-267.

[5] M. Brion and R. Joshua, Equivariant intersection cohomology of semi-stable points, Amer. J. Math. 118 (1996), no. 3, 595-610.

[6] M. Demazure, Invariants symétriques entiers des groupes de Weyl et torsion, Invent. Math. 21 (1973), 287-301 (French).

[7] D. Edidin and W. Graham, Equivariant intersection theory, Invent. Math. 131 (1998), no. 3, 595-634.

[8] Localization in equivariant intersection theory and the Bott residue formula, Amer. J. Math. 120 (1998), no. 3, 619-636.

[9] D. Edidin, A. Geraschenko, and M. Satriano, There is no degree map for O-cycles on Artin stacks, Transform. Groups 18 (2013), no. 2, 385-389.

[10] G. Ellingsrud and S. A. Strømme, On the Chow ring of a geometric quotient, Ann. of Math. (2) 130 (1989), no. 1, 159-187.

[11] W. Fulton, Intersection theory, 2nd ed., Ergebnisse der Mathematik und ihrer Grenzgebiete. 3. Folge. A Series of Modern Surveys in Mathematics [Results in Mathematics and Related Areas. 3rd Series. A Series of Modern Surveys in Mathematics], vol. 2, Springer-Verlag, Berlin, 1998.

[12] D. Mumford, J. Fogarty, and F. Kirwan, Geometric invariant theory, 3rd ed., Ergebnisse der Mathematik und ihrer Grenzgebiete (2) [Results in Mathematics and Related Areas (2)], vol. 34, Springer-Verlag, Berlin, 1994.

[13] V. Guillemin and J. Kalkman, The Jeffrey-Kirwan localization theorem and residue operations in equivariant cohomology, J. Reine Angew. Math. 470 (1996), 123-142.

[14] H. Gillet, Intersection theory on algebraic stacks and Q-varieties, Proceedings of the Luminy conference on algebraic $K$-theory (Luminy, 1983), 1984, pp. 193-240.

[15] W. H. Hesselink, Uniform instability in reductive groups, J. Reine Angew. Math. 303/304 (1978), 74-96. 
[16] Desingularizations of varieties of nullforms, Invent. Math. 55 (1979), no. 2, 141-163.

[17] J. E. Humphreys, Linear algebraic groups, Springer-Verlag, New York, 1975. Graduate Texts in Mathematics, No. 21.

[18] L. C. Jeffrey and F. C. Kirwan, Localization for nonabelian group actions, Topology 34 (1995), no. 2, 291-327.

[19] S. Keel and S. Mori, Quotients by groupoids, Ann. of Math. (2) 145 (1997), no. 1, 193-213.

[20] G. R. Kempf, Instability in invariant theory, Ann. of Math. (2) 108 (1978), no. 2, 299-316.

[21] G. Kempf and L. Ness, The length of vectors in representation spaces, Algebraic geometry (Proc. Summer Meeting, Univ. Copenhagen, Copenhagen, 1978), Lecture Notes in Math., vol. 732, Springer, Berlin, 1979, pp. $233-243$.

[22] F. C. Kirwan, Cohomology of quotients in symplectic and algebraic geometry, Mathematical Notes, vol. 31, Princeton University Press, Princeton, NJ, 1984.

[23] Partial desingularisations of quotients of nonsingular varieties and their Betti numbers, Ann. of Math. (2) 122 (1985), no. 1, 41-85.

[24] S. Martin, Symplectic quotients by a nonabelian group and by its maximal torus, arXiv:math/0001002v1 [math.SG] (2000), unpublished.

[25] P.-E. Paradan, The moment map and equivariant cohomology with generalized coefficients, Topology 39 (2000), no. 2, 401-444.

[26] Z. Reichstein, Stability and equivariant maps, Invent. Math. 96 (1989), no. 2, 349-383.

[27] C. S. Seshadri, Geometric reductivity over arbitrary base, Advances in Math. 26 (1977), no. 3, 225-274.

[28] R. Steinberg, The isomorphism and isogeny theorems for reductive algebraic groups, J. Algebra 216 (1999), no. 1, 366-383.

[29] B. Totaro, The Chow ring of a classifying space, Algebraic $K$-theory (Seattle, WA, 1997), Proc. Sympos. Pure Math., vol. 67, Amer. Math. Soc., Providence, RI, 1999, pp. 249-281.

[30] M. Vergne, A note on the Jeffrey-Kirwan-Witten localisation formula, Topology 35 (1996), no. 1, 243-266.

[31] A. Vistoli, Intersection theory on algebraic stacks and on their moduli spaces, Invent. Math. 97 (1989), no. 3, 613-670.

[32] E. Witten, Two-dimensional gauge theories revisited, J. Geom. Phys. 9 (1992), no. 4, 303-368. 